\title{
Radon, Thoron and Progeny Measured in Urban Health Centres and the Resulting Radiation Doses to Doctors, Nurses and Patients from the Inhalation of Air
}

\author{
M. A. Misdaq ${ }^{1, *}$, A. Matrane ${ }^{1,2}$, J. Ouguidi ${ }^{1}$ \\ ${ }^{1}$ Nuclear Physics and Techniques Laboratory, Faculty of Sciences Semlalia, University of Cadi Ayyad, Marrakech, Morocco (URAC-15 \\ Research Unit Associated to the CNRST, Rabat, Morocco) \\ ${ }^{2}$ Nuclear Medicine Service, Mohamed VI University Hospital Centre, Faculty of Medicine and Pharmacy, University of Cadi Ayyad, \\ Marrakech, Morocco
}

Email address:

misdaq@uca.ma (M. A. Misdaq)

\section{To cite this article:}

M. A. Misdaq, A. Matrane, J. Ouguidi. Radon, Thoron and Progeny Measured in Urban Health Centres and the Resulting Radiation Doses to Doctors, Nurses and Patients from the Inhalation of Air. American Journal of Environmental Protection. Vol. 4, No. 3, 2015 , pp. $139-151$. doi: $10.11648 /$ j.ajep.20150403.15

\begin{abstract}
Doctors and nurses spent about 8 hours a day inside urban health centres examining a large number of patients. To assess radiation dose due to the attached and unattached fractions of the short-lived alpha-emitting radon decay products from the inhalation of air by working personnel and patients, concentrations of these radionuclides as well as those of radon and thoron gases were measured in indoor air of different health centres in the city of Marrakech (Morocco) by means of CR-39 and LR-115 type II solid state nuclear track detectors (SSNTDs). Committed equivalent doses per hour of exposure due to the attached and unattached fractions of ${ }^{218} \mathrm{Po}$ and ${ }^{214} \mathrm{Po}$ radon short-lived progeny were evaluated in different tissues of the respiratory tract of individuals from the inhalation of air inside the studied health centres. The influence of the activity of the attached and unattached fractions of ${ }^{218} \mathrm{Po}$ and ${ }^{214} \mathrm{Po}$ and mass of the tissue on the committed equivalent doses per hour of exposure was investigated. Annual committed effective doses due to the attached and unattached fractions of ${ }^{218} \mathrm{Po}$ and ${ }^{214} \mathrm{Po}$ radon short-lived progeny from the inhalation of air by doctors, nurses and patients inside the studied hospitals were determined. A maximum value of $7.1 \mathrm{mSv} \mathrm{y}^{-1}$ was found for doctors working 40 hours per week.
\end{abstract}

Keywords: Radon, Thoron and Their Progenies, Attached and Unattached Fractions, Health Centres,

Nuclear Track Detectors, Human Respiratory Tract, Radiation Dose Assessment

\section{Introduction}

Humans are continuously exposed in homes and workplaces to some ionising radiation in form of alpha- and beta- and gamma-photons emitted by radon, thoron and their corresponding decay products coming from building materials, thermal water and air pollution (cigarette smoke, fly ashes and dusts) [1-5]. Radon and its short-lived progeny in dwellings and workplaces represent the main source of public exposure from natural radiation. They contribute to nearly $50 \%$ of the global effective dose to population [6]. Lung cancer hazard from inhalation exposure to radon is due to alpha-dose deposited by short-lived radon progeny. Being inert gases, the main characteristic of radon $\left({ }^{222} \mathrm{Rn}\right)$ and thoron $\left({ }^{220} \mathrm{Rn}\right)$ among other natural radioelements is the fact that their behaviour is not affected by chemical processes. In addition, their concentration levels depend strongly on geological and geophysical conditions, as well as on atmospheric influences such as barometric pressure and rainfall. Formed as a result of ${ }^{238} \mathrm{U}$ and ${ }^{232} \mathrm{Th}$ natural radioactive series in the earth's crust they are free to move through soil pores and rock fractures; then to escape into the atmosphere. Once in the air atmosphere, the ${ }^{222} \mathrm{Rn}$ and ${ }^{220} \mathrm{Rn}$ atoms decay producing isotopes of polonium, lead, and bismuth- also thallium for ${ }^{220} \mathrm{Rn}$. These elements are heavy metals chemically very active, which may exist briefly as ions/ or free atoms before forming molecules in condensed phase or attached to airborne dust particles, typically to those with a sub-micron range sizes, forming radioactive aerosols. A variable proportion of airborne ${ }^{222} \mathrm{Rn}$ and ${ }^{220} \mathrm{Rn}$ decay products remains unattached and is referred as the airborneunattached fraction. This fraction may be inhaled and 
deposited in the respiratory tract, in which they release all their alpha-emissions. The particle distribution of the aerosolattached fraction in the inhaled air also influences the dose to the airways, because particles of different sizes deposit preferentially in different areas of the respiratory tract.In controlling radiation exposure for the working personnel and patients inside hospitals, it is necessary to measure the concentrations of radon, thoron and their progenies in the air of these locations. ${ }^{222} \mathrm{Rn}$ concentration was measured in dwellings and workplaces in different countries by using LR115 type films[7], Pylon AB-5 scintillometer with a continuous passive radon detector [8], AlphaGUARDPQ 2000PRO [8] and Radim 2P and Radim 3P with a semiconductor detector $[8,9]$.

In the work described here, a technique based on using CR-39 and LR-115 type II SSNTDs was used for measuring the concentration of radon, thoron and their progenies inside different urban health centres. Annual committed effective doses due to the inhalation of radon short- lived decay products by doctors, nurses and patientsinside the studied health centres were also evaluated.

\section{Materials and Methods}

\subsection{Description of the Studied Health Centres}

The studied health centres are situated in different districts of the city of Marrakech (Morocco) in which thousands of patients received health cares all over the year. They are generally built with concrete, cement, bricks and granite rocks as shown in Table 1. The surface (S) to volume (V) ratio is equal to $1.5 \mathrm{~m}^{-1}$ for doctors' and nurses' offices and $2.0 \mathrm{~m}^{-1}$ for patients' rooms for the studied urban health centres.

Table 1. Description of the studied health centres

\begin{tabular}{|c|c|c|c|}
\hline \multirow{2}{*}{ Health centre } & \multicolumn{3}{|l|}{ Building materials } \\
\hline & Walls & Floor & Ceiling \\
\hline $\mathrm{HC} 1$ & Concrete, cement & Concrete, cement, granite & Concrete, cement \\
\hline $\mathrm{HC} 2$ & Concrete, cement & Concrete, cement, granite & Concrete, cement \\
\hline $\mathrm{HC} 3$ & Concrete, cement & Concrete, cement, granite & Concrete, cement \\
\hline $\mathrm{HC} 4$ & Bricks, cement & Concrete, cement, granite & Concrete, cement \\
\hline $\mathrm{HC} 5$ & Cement, granite & Concrete, cement, granite & Zinc plaques, cement \\
\hline HC6 & Bricks, cement & Concrete, cement, granite & Concrete, cement \\
\hline $\mathrm{HC} 8$ & Bricks, cement & Concrete, cement, granite & Concrete, cement \\
\hline HC9 & Bricks, cement & Concrete, cement, granite & Concrete, cement \\
\hline $\mathrm{HC} 10$ & Bricks, cement & Concrete, cement, granite & Concrete, cement \\
\hline
\end{tabular}

\subsection{Determination of Alpha- and Beta-Activities Per Unit Volume Due to Radon, Thoron and Their Decay Products in the Air of Urban Health Centres}

The alpha-activities of ${ }^{222} \mathrm{Rn},{ }^{220} \mathrm{Rn}$ and their decay products were measured using the following types of solid state nuclear track detectors (SSNTDs):

(i) CR-39 discs, $2 \mathrm{~cm}$ in radius and $500 \mu \mathrm{m}$ thick, manufactured by Pershore Mouldings Ltd, United Kingdom;

(ii) LR-115 type II discs, $2 \mathrm{~cm}$ in radius, comprising 12 $\mu \mathrm{m}$ of cellulose nitrate on a $100 \mu \mathrm{m}$ thick polyester base, manufactured by Kodak Pathé, France, and marketed by Dosirad, France.

The detectors were hung in different doctors' and nurses' offices and patients' rooms inside health centres in the city of Marrakech (Morocco) for 24 hours in May 2014(Figure 1). During the exposure time, $\alpha$-particles emitted by radon, thoron and their progenies bombarded the SSNTD films. After the irradiation, the exposed SSNTDs were etched in two $\mathrm{NaOH}$ solutions: one was of 2.5 normality at $60^{\circ} \mathrm{C}$ during 2 hours for the LR-115 II films, andthe other of 6.25 normality at $70^{\circ} \mathrm{C}$ for 7 hours for the CR-39 detectors [10]. After chemical treatment, the track densities registered on the CR-39 and LR-115 II SSNTDs were determined by means of an ordinary microscope with magnification 40x. Backgrounds of the CR-39 and LR-115II SSNTDs have been evaluated by placing five unexposed films inside small well closed plastic pockets during 24 hours in each location of the studied hospitals and counting the resulting track density rates. The average track density rates were found to be equal to $\rho_{G}^{C R}=(1.58 \pm 0.01) 10^{-6}$ tracks cm $\mathrm{cs}^{-2}$ and $\rho_{G}^{L R}=(0.535 \pm$ $0.005) 10^{-6}$ tracks cm ${ }^{-2} \mathrm{~s}^{-1}$, respectively. The reproducibility of the method was checked by placing four couples of CR-39 and LR-115 type II SSNTDs at the same room in the studied health centres for 24 hours. The distance between two successive couples of detectors is of $30 \mathrm{~cm}$. The average track density rates registered on the CR-39 and LR-115 type II SSNTDs were evaluated. The relative uncertainty of the average track density rate determination is smaller than $1 \%$.For the experimental etching conditions, the residual thickness of the LR-115 type II SSNTD is $5 \mu \mathrm{m}$ which corresponds to the lower $\left(\mathrm{E}_{\min }=1.6 \mathrm{MeV}\right)$ and upper $\left(E_{\max }=4.7 \mathrm{MeV}\right)$ energy limits for the registration of tracks of alpha-particles in the LR-115 II films [11]. All alpha-particles emitted by the radon and thoron series that reach the LR-115 II detector surface under an angle lower than its critical angle of etching, $\theta_{c}^{\prime}$ with a residual energy between $1.6 \mathrm{MeV}$ and 4.7 $\mathrm{MeV}$ are registered as bright track-holes. The CR-39 detector is sensitive to all alpha-particles reaching its surface under an angle smaller than its critical angle of etching $\theta_{c}$. The critical angles of etching $\theta_{c}^{\prime}$ and $\theta_{c}$ were calculated using a method described in detail by Misdaq et al. [12]. 


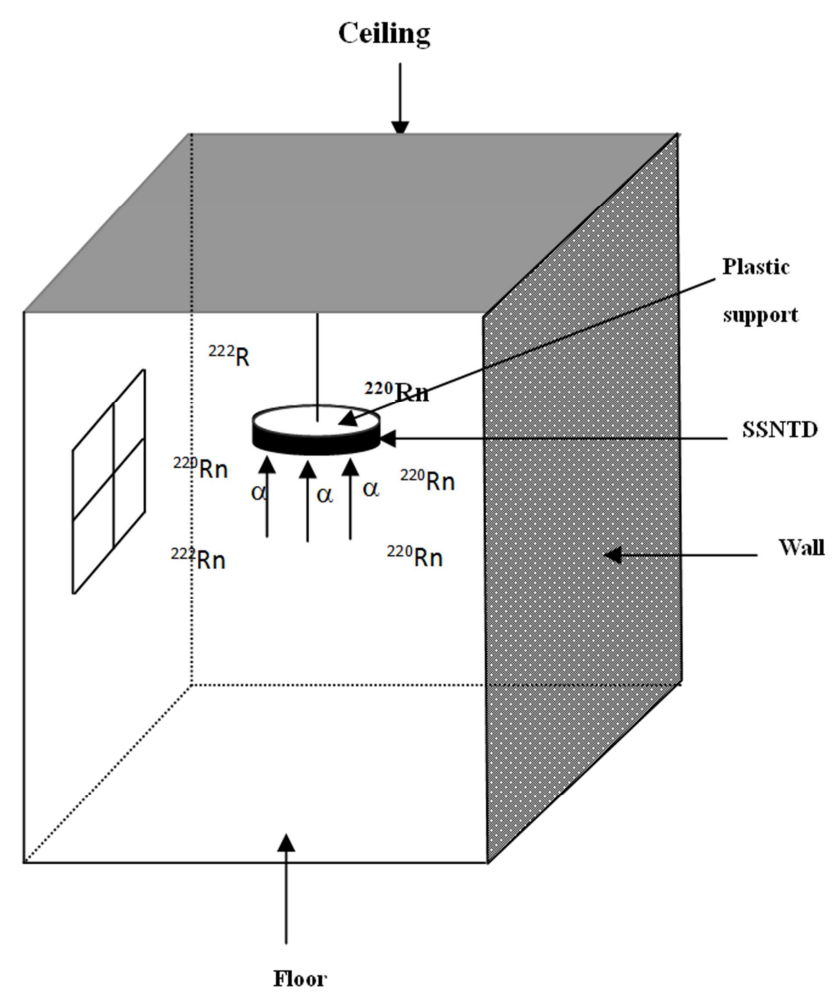

Fig. 1. Sketch of the arrangement of the CR-39 and LR-115 type II solid state nuclear track detectors inside a room in a health centre. The detectors are placed at a distance of $80 \mathrm{~cm}$ from the ceiling and $220 \mathrm{~cm}$ from the floor.

The global track density rates (tracks $\mathrm{cm}^{-2} \mathrm{~s}^{-1}$ ), due to $\alpha$ particles emitted by the ${ }^{222} \mathrm{Rn}$ (three $\alpha$-emitters) and ${ }^{220} \mathrm{Rn}$ (four $\alpha$-emitters) series inside a location, registered on the CR-39 $\left(\rho_{G}^{C R}\right)$ and LR-115 II $\left(\rho_{G}^{L R}\right)$ detectors, after subtracting the corresponding backgrounds, were obtained as described by [13]:

$\rho_{G}^{C R}=\frac{\pi q^{2}}{2 S_{d}^{\prime}}\left\{\sum_{1}^{3} A_{c}(j) K_{j} \mathcal{E}_{j}^{C R} R_{j}+\sum_{1}^{4} A_{c}(j) K_{j}^{\prime}{\left.\mathcal{E}^{\prime}{ }_{j}{ }^{C R} R^{\prime}{ }_{j}\right\}}^{\prime}\right.$

and

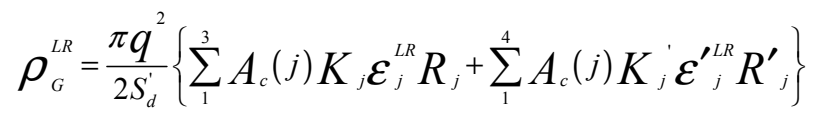

where $q$ is the radius of the detector films $(2 \mathrm{~cm}), S_{d}$ and $S_{d}^{\prime}$ are respectively the surface areas of the CR-39 and LR-115 II films, $A_{c}(j)\left(B q c^{-3}\right)$ is the alpha-activity of the $j^{\text {thalpha- }}$ emitter, $R_{j}$ and $R_{j}^{\prime}$ are the ranges in air of an alpha-particle of index $j$ and initial energy $E_{j}$ emitted by the nuclei of the ${ }^{222} \mathrm{Rn}$ and ${ }^{220} \mathrm{Rn}$ series inside the air of the studied cave, respectively, $K_{j}$ and $K_{j}^{\prime}$ are, respectively, the branching ratios corresponding to the disintegration of the nuclei of the ${ }^{222} \mathrm{Rn}$ and ${ }^{220} \mathrm{Rn}$ series and $\varepsilon_{j}^{C R}, \varepsilon_{j}^{\prime C R}, \varepsilon_{j}^{L R}$ and $\varepsilon_{j}^{\prime L R}$ are respectively the detection efficiencies of the CR-39 and LR115 II detectors for the emitted $\alpha$-particles [13]. The first terms (right of Equations (1) and (2)) correspond to the number of alpha-particles emitted by the radon series, when the second terms correspond to the number of alpha-particles emitted by the thoron group.

The relationship between the activity concentrations of the unattached fraction $A_{c, u}(j-1)$ and attached fraction $A_{c, a}(j-1)$ of a $(\mathrm{j}-1)^{\text {th }}$ radionuclide and those of its $\mathrm{j}^{\text {th }}$ unattached progeny $A_{c, u}(j)$ and attached progeny $A_{c, a}(j)$ is given by the following [14]:

$$
\begin{gathered}
A_{c}(j)=A_{c, a}(j)+A_{c, u}(j) \\
A_{c, u}(j)=\frac{\lambda_{j} A_{c, u}(j-1)+r_{j-1} \lambda_{j} A_{c, a}(j-1)}{V+\lambda_{j}+q^{u}+X}
\end{gathered}
$$

and

$$
A_{c, a}(j)=\frac{\left(1-r_{j-1}\right) \lambda_{j} A_{c, a}(j-1)+X A_{c, u}(j)}{V+\lambda_{j}+q^{a}}
$$

where $\lambda_{j}\left(\mathrm{~s}^{-1}\right)$ is the decay constant of the $\mathrm{j}^{\text {th }}$ radon or thoron progeny, $r_{j}$ is the recoil factor of the aerosol-attached radon or thoron progeny $\mathrm{j}, \mathrm{V}$ is the ventilation rate inside a location which is measured by using a $\mathrm{CO}_{2}$ tracer method and was found to be in the range of $0.26-0.57 \mathrm{~h}^{-1}$ ) (Table 2), $\mathrm{X}$ is the mean attachment rate [14], $\mathrm{q}^{\mathrm{u}}$ is the plate-out rate of the unattached radon or thoron daughters [14] and $\mathrm{q}^{\mathrm{a}}$ is the plateout rate of the aerosol-attached radon or thoron progenies [14].

The values of $\mathrm{j}$ for radon and its progeny are:

- ${ }^{222} \mathrm{Rn}: \mathrm{j}=0$;

- ${ }^{218} \mathrm{Po}: \mathrm{j}=1$;

- ${ }^{214} \mathrm{~Pb}: \mathrm{j}=2$;

- ${ }^{214} \mathrm{Bi}: \mathrm{j}=3$;

- ${ }^{214} \mathrm{Po}: \mathrm{j}=4$.

The corresponding values for ${ }^{220} \mathrm{Rn}$ and its progeny are:

- ${ }^{220} \mathrm{Rn}: \mathrm{j}=0$;

- ${ }^{216} \mathrm{Po} / \mathrm{j}=1$;

- ${ }^{212} \mathrm{~Pb}: \mathrm{j}=2$;

- ${ }^{212} \mathrm{Bi}: \mathrm{j}=3$;

- ${ }^{212} \mathrm{Po}: \mathrm{j}=4$.

Since ${ }^{222} \mathrm{Rn}$ and ${ }^{220} \mathrm{Rn}$ are gaseous, all of the activity is unattached, that is:

- $A_{c, u}(0)=A_{c}(0)$

- $A_{c, a}(0)=0$.

The recoil factors for the attached ${ }^{222} \mathrm{Rn}$ and ${ }^{220} \mathrm{Rn}$ progeny are as follows [15]:

- $\mathrm{r}_{1}=\mathrm{r}_{4}=0.8$

- $\mathrm{r}_{2}=\mathrm{r}_{3}=0$.

The plate-out rate of the unattached radon or thoron daughters' $\mathrm{q}^{\mathrm{u}}$ is equal to $22.5 \mathrm{~h}^{-1}$ for doctors' and nurses' offices and $30.0 \mathrm{~h}^{-1}$ for patients' rooms in the studied health centres. The plate-out rate of the aerosol-attached radon or thoron progenies $\mathrm{q}^{\mathrm{a}}$ is equal to $0.15 \mathrm{~h}^{-1}$ for doctors' and nurses' offices and $0.2 \mathrm{~h}^{-1}$ for patients' rooms in the studied health centres. The mean attachment rate $\mathrm{X}$ was adjusted to get values of the radon and thoron concentrations comparable to those obtained by using a method previously developed by Misdaq et al. [13], within the uncertainty interval, for doctors' 
and nurses offices and patients' rooms inside the studied health centres (Table 3 ).

\subsection{Evaluation of Annual Committed Effective Doses Due to the Inhalation of Radon Short-Lived Decay Products in the Respiratory Tract of Working Personnel and Patients Inside a Health Centre}

In terms of the International Commission on Radiological Protection [16,17], there are ten compartments in the thoracic region of the human respiratory tract numbered from 1 to 10and, respectively, named $\mathrm{AI}_{1}, \mathrm{AI}_{2}, \mathrm{AI}_{3}, \mathrm{bb}_{1}, \mathrm{bb}_{2}, \mathrm{bb}_{\text {seq }}, \mathrm{BB}_{1}$,
$\mathrm{BB}_{2}, \mathrm{BB}_{\text {seq }}$ and $\mathrm{LN}_{\mathrm{TH}}$. The extrathoracic region contains four compartments numbered from 11 to 14 and, respectively named $\mathrm{ET}_{2}, \mathrm{ET}_{\text {seq }}, \mathrm{LN}_{\mathrm{ET}}$ and $\mathrm{ET}_{1}$. As radon is inert, nearly all the gas inhaled is subsequently exhaled. However, when inhaled, the radon short-lived progeny are assumed to be attached to particles of an activity median aerodynamic diameter (AMAD) of $200 \mathrm{~nm}$ with a geometric standard deviation $\sigma_{\mathrm{g}}=2.35$ [16] in indoor air. The rate of change of the alpha-activity of the attached fraction of $\mathrm{a}^{j^{\text {th }}}$ radon decay product in a compartment $i$ of the respiratory tract [16] at any time is given by:

$$
\frac{d A^{i}\left(j^{\prime}\right)}{d t}=F_{d}(i) I_{0}\left(j^{\prime}\right)+\sum_{n} \lambda_{n, i} A_{c, a}^{n}\left(j^{\prime}\right)-\left(\sum_{n} \lambda_{i, n}+\lambda_{j^{\prime}}\right) A_{c, a}^{i}\left(j^{\prime}\right)
$$

where $F_{d}(i)$ is the fractional deposition in the compartment $i$ of the respiratory tract of individuals[16], $I_{0}\left(j^{\prime}\right)=B A_{c, a}\left(j^{\prime}\right) . \mathrm{B}$ is the average breathing rate for workers and patients in a health center. $A_{c, a}\left(j^{\prime}\right)\left(B q \cdot m^{-3}\right)$ is the alpha-activity of the attached fraction of a $\mathrm{j}^{\text {th }}$ radon decay product in a health centre atmosphere, $\lambda_{\mathrm{n}, \mathrm{i}}=\mathrm{m}_{\mathrm{n}, \mathrm{i}}+\mathrm{S}_{\mathrm{s}}$ where $\mathrm{m}_{\mathrm{n}, \mathrm{i}}$ is the clearance rate from region $\mathrm{n}$ to region $\mathrm{i}$ due to particle transport and $\mathrm{S}_{\mathrm{s}}$ is the clearance rate due to particle absorption into blood [16]. The rate of absorption of a material into blood is the same in all regions of the respiratory tract, except in the anterior nasal passages $\left(\mathrm{ET}_{1}\right)$, where no absorption occurs [16]. $\lambda_{n, i}=0$ for $\mathrm{i}=1,2,3,5,6,8,9,12$ and $14[16], \lambda_{i, n}=m_{i, n}+S_{s}$ where $m_{i, n}$ is the clearance rate from region $\mathrm{i}$ to region $\mathrm{n}$ due to particle transport [16] and $\lambda_{j^{\prime}}$ is the radioactive constant of the $\mathrm{j}^{\text {th }}$ radon decay product.

Alpha-activities corresponding to the attached fraction of the $\mathrm{j}^{\text {th }}$ radon decay product in each of the 1-14 compartments of the respiratory tract as functions of time are obtained by solving Equation [6]. Alpha-equivalent dose rate $\left(\mathrm{Sv} \mathrm{s}^{-1}\right)$ in a tissue $\mathrm{T}$ of the respiratory tract of an individual due to the inhalation of the attached fraction of the $\mathrm{j}^{\mathrm{j}^{\text {th }}}$ radon daughter is given by:

$$
\dot{H}_{T}^{a}\left(j^{\prime}\right)(t)=A_{c, a}^{T}\left(j^{\prime}\right)(t) D_{S P}^{T}\left(j^{\prime}\right) W_{R}
$$

where $A_{c, a}^{T}\left(j^{\prime}\right)(t)(\mathrm{Bq})$ is the alpha-activity of the attached fraction of the $\mathrm{j}^{\text {th }}$ radon decay product in the tissue $\mathrm{T}$ of the respiratory tract, $D_{S P}^{T}\left(j^{\prime}\right)$ is the specific alpha-dose (Gy) deposited by alpha-particles emitted by $1 \mathrm{~Bq}$ of the $\mathrm{j}^{\mathrm{j}^{\text {th }}}$ radon daughter inside the tissue $\mathrm{T}$ and $\mathrm{W}_{\mathrm{R}}$ is the radiation weighting factor which is equal to 20 for alpha-particles [18].

The $D_{S P}^{T}\left(j^{\prime}\right)$ specific alpha-dose is given by:

$$
D_{S P}^{T}\left(j^{\prime}\right)=k \frac{K_{j^{\prime}}, R_{j^{\prime}} S_{j^{\prime}}}{m_{T}}
$$

where $\mathrm{m}_{\mathrm{T}}$ is the mass of the target tissue $\mathrm{T}(18), \mathrm{K}_{\mathrm{j}}$ is the branching ratio, $R_{j^{\prime}}$ is the range of the alpha-particle emitted by the $j^{\text {th }}$ radon decay product, $S_{j^{\prime}}$ is the stopping power of the tissue $\mathrm{T}$ for the emitted alpha-particle and $\mathrm{k}=1.610^{-10}$ is a conversion factor. $R_{j}$ and $S_{j}$ were calculated by using the TRIM program [19] (using the elemental chemical composition of tissues given in the ICRP publication 89 [20]).

The equivalent dose in the tissue $\mathrm{T}$ of the respiratory tract for the attached fraction of the $\mathrm{j}$ ' radon decay product is given by:

$$
H_{T}^{a}\left(j^{\prime}\right)=\int_{0}^{t^{\prime}} \dot{H}_{T}^{a}\left(j^{\prime}\right)(t) d t
$$

where $t_{\mathrm{e}}{ }^{\prime}$ is the exposure time of the tissue $\mathrm{T}$.

The rate of change of the alpha-activity of the unattached fraction of the $\mathrm{j}^{\text {th }}$ radon decay product $(\mathrm{AMAD}=1 \mathrm{~nm})(14) \mathrm{in}$ a compartment $i$ of the respiratory tract [16] at any time is given by:

$$
\frac{d A_{c, u}^{i}\left(j^{\prime}\right)}{d t}=F_{d}(i) I_{0}\left(j^{\prime}\right)+\sum \lambda_{n, i} A_{c, u}^{n}\left(j^{\prime}\right)-\left(\sum_{n} \lambda_{i, n}+\lambda_{j^{\prime}}\right) A_{c, u}^{i}\left(j^{\prime}\right)
$$

where $F_{d}(i)$ is the fractional deposition in the compartment $i$ of the respiratory tract of individuals(16), $I_{0}\left(j^{\prime}\right)=B A_{c, u}\left(j^{\prime}\right) . \mathrm{B}$ is the average breathing rate for working personnel and patients in a health centre, $A_{c, u}\left(j^{\prime}\right)(B q$ $\mathrm{m}^{-3}$ ) is the alpha-activity of the unattached fraction of the $\mathrm{j}^{\text {th }}$ radon decay product in a health centre atmosphere.

Alpha-activities corresponding to the unattached fraction of the $j^{\text {th }}$ radon decay product in each of the 1-14 compartments of the respiratory tract as functions of time are obtained by solving Equation [10].

Alpha-equivalent dose rate $\left(\mathrm{Sv} \mathrm{s}^{-1}\right)$ in a tissue $\mathrm{T}$ of the respiratory tract of an individual due to the inhalation of the unattached fraction of the $j^{\text {th }}$ radon daughter is given by: 


$$
\dot{H}_{T}^{u}\left(j^{\prime}\right)(t)=A_{c, u}^{T}\left(j^{\prime}\right)(t) D_{S P}^{T}\left(j^{\prime}\right) W_{R}
$$

where $A_{c, u}^{T}\left(j^{\prime}\right)(t)(\mathrm{Bq})$ is the alpha-activity of the unattached fraction of a $\mathrm{j}^{\text {th }}$ radon decay product in the tissue $\mathrm{T}$ of the respiratory tract.

The equivalent dose in the tissue $\mathrm{T}$ of the respiratory tract for the unattached fraction of the $\mathrm{j}^{\prime}$ radon decay product is given by:

$$
H_{T}^{u}\left(j^{\prime}\right)=\int_{0}^{t^{\prime}} \dot{H}_{T}^{u}\left(j^{\prime}\right)(t) d t
$$

Regional doses, weighted with factors assigned for the partition of radiation detriment, are summed to give a value of committed equivalent dose for the thoracic $\mathrm{H}_{\mathrm{TH}}\left(\mathrm{j}^{\prime}\right)$ and extrathoracic $\mathrm{H}_{\mathrm{ET}}\left(\mathrm{j}^{\prime}\right)$ regions. Indeed, we have:

$$
\begin{aligned}
& H_{T H}\left(j^{\prime}\right)=A_{B B}\left(H_{B B}^{a}\left(j^{\prime}\right)+H_{B B}^{u}\left(j^{\prime}\right)\right)+A_{b b}\left(H_{b b}^{a}\left(j^{\prime}\right)+H_{b b}^{u}\left(j^{\prime}\right)\right)+ \\
& A_{A I}\left(H_{A I}^{a}\left(j^{\prime}\right)+H_{A I}^{u}\left(j^{\prime}\right)\right)+A_{L N T H}\left(H_{L N T H}^{a}\left(j^{\prime}\right)+H_{L N T H}^{u}\left(j^{\prime}\right)\right)
\end{aligned}
$$

and

$$
\begin{aligned}
& H_{E T}\left(j^{\prime}\right)=A_{E T 1}\left(H_{E T 1}^{a}\left(j^{\prime}\right)+H_{E T 1}^{u}\left(j^{\prime}\right)\right)+A_{E T 2}\left(H_{E T 2}^{a}\left(j^{\prime}\right)+\right. \\
& \left.H_{E T 2}^{u}\left(j^{\prime}\right)\right)+A_{L N E T}\left(H_{L N E T}^{a}\left(j^{\prime}\right)+H_{L N E T}^{u}\left(j^{\prime}\right)\right)
\end{aligned}
$$

Where

$$
E^{u}=0.12\left[H_{T H}^{u}\left({ }^{218} P o\right)+H_{T H}^{u}\left({ }^{214} P o\right)\right]+0.025\left[H_{E T}^{u}\left({ }^{218} P o\right)+H_{E T}^{u}\left({ }^{214} P o\right)\right]
$$

is the committed effective dose due to the unattached fractions of the ${ }^{218} \mathrm{Po}$ and ${ }^{214} \mathrm{Po}$ radon progeny and

$$
E^{a}=0.12\left[H_{T H}^{a}\left({ }^{218} P o\right)+H_{T H}^{a}\left({ }^{214} P o\right)\right]+0.025\left[H_{E T}^{a}\left({ }^{218} P o\right)+H_{E T}^{a}\left({ }^{214} P o\right)\right]
$$

is the committed effective dose due to the attached fractions of the ${ }^{218} \mathrm{Po}$ and ${ }^{214} \mathrm{Po}$ radon progeny.

\section{Results and Discussion}

\subsection{Alpha- and Beta-Activities per Unit Volume Due to ${ }^{222} \mathrm{Rn},{ }^{220} \mathrm{Rn}$ and Their Progenies in the Air of Different

\begin{tabular}{|c|c|c|c|c|c|c|c|c|c|}
\hline \multirow[t]{2}{*}{ Health centre } & \multicolumn{3}{|c|}{ Doctor's office } & \multicolumn{3}{|c|}{ Nurse's office } & \multicolumn{3}{|c|}{ Patients' room } \\
\hline & $V\left(h^{-1}\right)$ & $\begin{array}{l}A_{c}\left({ }^{222} R n\right) \\
\left(B q \cdot m^{-3}\right)\end{array}$ & $\begin{array}{l}A_{c}\left({ }^{220} R n\right) \\
\left(B q \cdot m^{-3}\right)\end{array}$ & $V\left(h^{-1}\right)$ & $\begin{array}{l}A_{c}\left({ }^{222} R n\right) \\
\left(B q \cdot m^{-3}\right)\end{array}$ & $\begin{array}{l}A_{c}\left({ }^{220} R n\right) \\
\left(B q \cdot m^{-3}\right)\end{array}$ & $V\left(h^{-1}\right)$ & $\begin{array}{l}A_{c}\left({ }^{222} R n\right) \\
\left(B q \cdot m^{-3}\right)\end{array}$ & $\begin{array}{l}\mathrm{A}_{\mathrm{c}}\left({ }^{220} \mathrm{Rn}\right) \\
\left(\mathrm{Bq} \cdot \mathrm{m}^{-3}\right)\end{array}$ \\
\hline $\mathrm{HC} 1$ & 0.50 & $74 \pm 5$ & $6.8 \pm 0.5$ & 0.45 & $78 \pm 5$ & $7.9 \pm 0.5$ & 0.40 & $88 \pm 6$ & $8.7 \pm 0.6$ \\
\hline $\mathrm{HC} 2$ & 0.50 & $74 \pm 5$ & $6.8 \pm 0.5$ & 0.46 & $77 \pm 5$ & $7.8 \pm 0.5$ & 0.46 & $78 \pm 5$ & $7.6 \pm 0.5$ \\
\hline $\mathrm{HC} 3$ & 0.29 & $131 \pm 10$ & $13 \pm 1$ & 0.28 & $138 \pm 10$ & $13.0 \pm 0.8$ & 0.30 & $127 \pm 9$ & $12.3 \pm 0.8$ \\
\hline $\mathrm{HC} 4$ & 0.26 & $165 \pm 11$ & $14 \pm 1$ & 0.40 & $88 \pm 6$ & $9.1 \pm 0.6$ & 0.48 & $76 \pm 5$ & $6.8 \pm 0.5$ \\
\hline $\mathrm{HC} 5$ & 0.30 & $125 \pm 9$ & $12.0 \pm 0.8$ & 0.39 & $90 \pm 6$ & $9.5 \pm 0.6$ & 0.48 & $76 \pm 5$ & $6.8 \pm 0.5$ \\
\hline HC6 & 0.31 & $119 \pm 9$ & $12.0 \pm 0.8$ & 0.34 & $105 \pm 8$ & $11.1 \pm 0.8$ & 0.37 & $95 \pm 6$ & $9.9 \pm 0.6$ \\
\hline $\mathrm{HC7}$ & 0.47 & $77 \pm 6$ & $7.4 \pm 0.5$ & 0.44 & $80 \pm 5$ & $8.0 \pm 0.5$ & 0.57 & $71 \pm 5$ & $6.2 \pm 0.4$ \\
\hline HC8 & 0.42 & $84 \pm 6$ & $8.4 \pm 0.6$ & 0.46 & $78 \pm 5$ & $7.6 \pm 0.5$ & 0.53 & $73 \pm 5$ & $6.4 \pm 0.4$ \\
\hline HC9 & 0.27 & $153 \pm 10$ & $13.3 \pm 0.8$ & 0.28 & $140 \pm 9$ & $13.1 \pm 0.8$ & 0.30 & $128 \pm 9$ & $12.6 \pm 0.8$ \\
\hline $\mathrm{HC} 10$ & 0.36 & $99 \pm 8$ & $10.3 \pm 0.8$ & 0.40 & $88 \pm 6$ & $9.5 \pm 0.6$ & 0.43 & $81 \pm 5$ & $8.2 \pm 0.6$ \\
\hline
\end{tabular} Urban Health Centres}

Alpha-activities per unit volume due to radon $\left({ }^{222} \mathrm{Rn}\right)$ and thoron $\left({ }^{220} \mathrm{Rn}\right)$ have been measured in May 2014 in the air of doctors' office, nurses' office and patients' room of various health centres in the city of Marrakech (Morocco).

Table 2. Data obtained for ${ }^{222} R n$ and ${ }^{220}$ Rn concentrations in doctor's offices, nurses' offices and patients' rooms of different health centres. $V$ is the ventilation rate. Given errors correspond to one-sigma uncertainties.

Data obtained are shown in Table 2. From the statistical error on track counting one can determine the error on track density rate and then evaluate the relative uncertainty of the radon and thoron determination which is of $8 \%$. It was noted 
that alpha-activities due to radon are higher than those due to thoron for the health centres studied. Indeed, due to its too short half-life $(55 \mathrm{~s})$ thoron $\left({ }^{220} \mathrm{Rn}\right)$ has a diffusion length in air smaller than that of radon $\left({ }^{222} \mathrm{Rn}\right)$ which has longer halflife $(3.82 \mathrm{~d})$. Note that since the studied health centres are practically built with the same materials (concrete, cement, granite and bricks), ${ }^{222} \mathrm{Rn}$ and ${ }^{220} \mathrm{Rn}$ concentrations increase when the ventilation rate inside the studied locations decreases. According to the ICRP publication 65 recommendations [21] the radon action level is situated between $500 \mathrm{~Bq} \mathrm{~m} \mathrm{~m}^{-3}$ and $1500 \mathrm{~Bq} \mathrm{~m}^{-3}$ for workplaces. The action level advised by the European Union [22], accepting the recommendations of the ICRP Publication 65 [21], is also the same: between $500 \mathrm{~Bq} \mathrm{~m}^{-3}$ and $1500 \mathrm{~Bq} \mathrm{~m}^{-3}$. In the United Kingdom, the Health and Safety Executive (HSE) has adopted a radon action level of $400 \mathrm{~Bq} \mathrm{~m} \mathrm{~m}^{-3}$ for workplaces based on advice from the National Radiological Protection
Board (NRPB) [23]. The USA has one reference level (150 $\mathrm{Bq} \mathrm{m}^{-3}$ ) which is applied for workplaces [24]. In Ireland, the advisory reference level is $200 \mathrm{~Bq} \mathrm{~m}^{-3}$ for workplaces [25]. In the European Union Directive 2013/59/EURATOM, the radon reference level is of $300 \mathrm{~Bq} \mathrm{~m}^{-3}$ in workplaces [26].In Morocco, the ICRP Publication 65 [21] recommendations are applied for workplaces.

Alpha-activities per unit volume air due to the attached and unattached fractions of the radon short-lived decay products were determined in the air of the studied health centres by using Equations (1)-(5). Data obtained are shown in Table 3. The relative uncertainty of the radon progenies determination is of $8 \%$. It is to be noted that the concentrations of the attached fraction of the radon shortlived decay products are clearly higher than those of the unattached fraction in the air of the studied health centres.

Table 3. Data obtained for the alpha-activities due to the unattached $\left(A_{c, u}\left({ }^{218} \mathrm{Po}\right)\right.$ and $\left.A_{c, u}\left({ }^{214} \mathrm{Po}\right)\right)$ and attached $\left(A_{c, a}\left({ }^{218} \mathrm{Po}\right)\right.$ and $\left.A_{c, a}\left({ }^{214} \mathrm{Po}\right)\right)$ fractions of the ${ }^{218} P$ o and ${ }^{214} P$ o radon short-lived progeny in nurses' office (a), doctors' office (b) and patients' room (c) of different health centres. Given errors correspond to one-sigma uncertainties.

Table 3 (a)

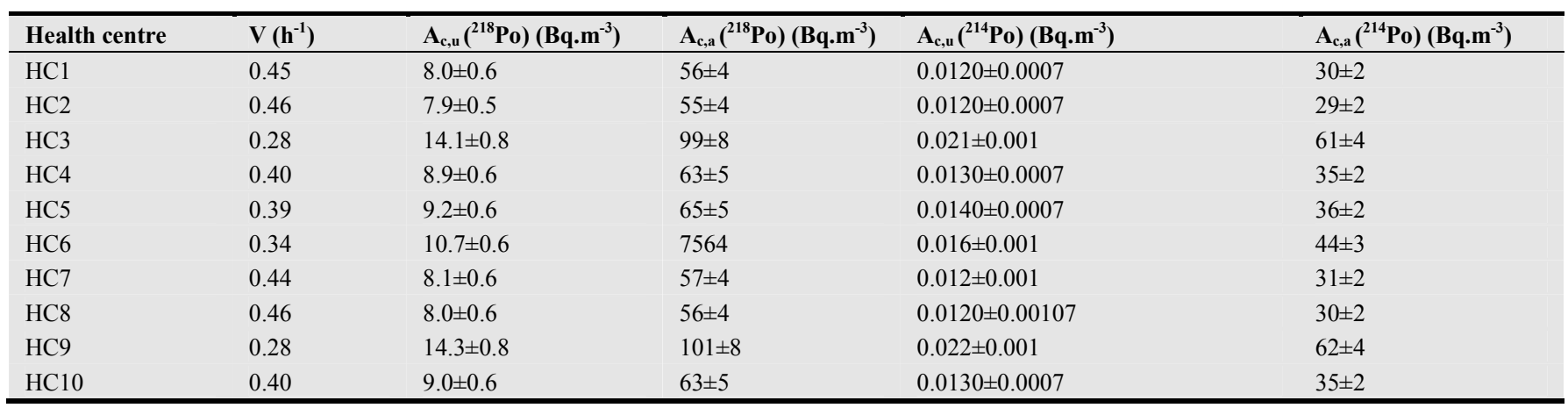

Table 3 (b)

\begin{tabular}{|c|c|c|c|c|c|}
\hline Health centre & $V\left(h^{-1}\right)$ & $\mathrm{A}_{\mathrm{c}, \mathrm{u}}\left({ }^{218} \mathrm{Po}\right)\left(\mathrm{Bq} \cdot \mathrm{m}^{-3}\right)$ & $\mathrm{A}_{\mathrm{c}, \mathrm{a}}\left({ }^{218} \mathrm{Po}\right)\left(\mathrm{Bq} \cdot \mathrm{m}^{-3}\right)$ & $A_{c, u}\left({ }^{214} P o\right)\left(B q \cdot m^{-3}\right)$ & $\mathrm{A}_{\mathrm{c}, \mathrm{a}}\left({ }^{214} \mathrm{Po}\right)\left(\mathrm{Bq} \cdot \mathrm{m}^{-3}\right)$ \\
\hline $\mathrm{HC} 1$ & 0.50 & $7.5 \pm 0.5$ & $53 \pm 4$ & $0.0110 \pm 0.0007$ & $27 \pm 2$ \\
\hline $\mathrm{HC} 2$ & 0.50 & $7.5 \pm 0.5$ & $53 \pm 4$ & $0.0110 \pm 0.0007$ & $27 \pm 2$ \\
\hline $\mathrm{HC} 3$ & 0.29 & $13.4 \pm 0.7$ & $95 \pm 7$ & $0.020 \pm 0.001$ & $57 \pm 4$ \\
\hline $\mathrm{HC} 4$ & 0.26 & $17.0 \pm 0.8$ & $119 \pm 9$ & $0.025 \pm 0.001$ & $74 \pm 5$ \\
\hline HC5 & 0.30 & $13.0 \pm 0.7$ & $90 \pm 7$ & $0.019 \pm 0.001$ & $54 \pm 4$ \\
\hline HC6 & 0.31 & $12.1 \pm 0.7$ & $86 \pm 6$ & $0.018 \pm 0.001$ & $51 \pm 4$ \\
\hline $\mathrm{HC7}$ & 0.47 & $7.8 \pm 0.5$ & $54 \pm 4$ & $0.0120 \pm 0.0007$ & $29 \pm 2$ \\
\hline $\mathrm{HC} 8$ & 0.42 & $8.5 \pm 0.5$ & $60 \pm 4$ & $0.0130 \pm 0.0007$ & $33 \pm 2$ \\
\hline $\mathrm{HC} 9$ & 0.27 & $15.6 \pm 0.8$ & $110 \pm 8$ & $0.024 \pm 0.001$ & $68 \pm 5$ \\
\hline $\mathrm{HC} 10$ & 0.36 & $10.0 \pm 0.6$ & $71 \pm 5$ & $0.0150 \pm 0.0007$ & $41 \pm 3$ \\
\hline
\end{tabular}

Table 3 (c)

\begin{tabular}{|c|c|c|c|c|c|}
\hline Health centre & $V\left(h^{-1}\right)$ & $\mathrm{A}_{\mathrm{c}, \mathrm{u}}\left({ }^{218} \mathrm{Po}\right)\left(\mathrm{Bq} \cdot \mathrm{m}^{-3}\right)$ & $\mathrm{A}_{\mathrm{c}, \mathrm{a}}\left({ }^{218} \mathrm{Po}\right)\left(\mathrm{Bq} \cdot \mathrm{m}^{-3}\right)$ & $A_{c, u}\left({ }^{214} P o\right)\left(B q \cdot m^{-3}\right)$ & $\mathrm{A}_{\mathrm{c}, \mathrm{a}}\left({ }^{214} \mathrm{Po}\right)\left(\mathrm{Bq} \cdot \mathrm{m}^{-3}\right)$ \\
\hline $\mathrm{HC} 1$ & 0.40 & $8.9 \pm 0.5$ & $63 \pm 4$ & $0.0130 \pm 0.0007$ & $35 \pm 2$ \\
\hline $\mathrm{HC} 2$ & 0.46 & $7.9 \pm 0.5$ & $56 \pm 4$ & $0.0120 \pm 0.0007$ & $30 \pm 2$ \\
\hline $\mathrm{HC} 3$ & 0.30 & $13.0 \pm 0.7$ & $92 \pm 7$ & $0.020 \pm 0.001$ & $55 \pm 4$ \\
\hline $\mathrm{HC} 4$ & 0.48 & $7.7 \pm 0.5$ & $54 \pm 4$ & $0.0120 \pm 0.0007$ & $28 \pm 2$ \\
\hline $\mathrm{HC} 5$ & 0.48 & $7.7 \pm 0.5$ & $54 \pm 4$ & $0.0120 \pm 0.0007$ & $28 \pm 2$ \\
\hline HC6 & 0.37 & $9.7 \pm 0.5$ & $68 \pm 4$ & $0.0150 \pm 0.0007$ & $39 \pm 2$ \\
\hline $\mathrm{HC7}$ & 0.57 & $7.2 \pm 0.5$ & $50 \pm 4$ & $0.0110 \pm 0.0007$ & $25 \pm 2$ \\
\hline $\mathrm{HC} 8$ & 0.53 & $7.4 \pm 0.5$ & $52 \pm 4$ & $0.0110 \pm 0.0007$ & $26 \pm 2$ \\
\hline HC9 & 0.30 & $13.0 \pm 0.7$ & $92 \pm 7$ & $0.020 \pm 0.001$ & $56 \pm 4$ \\
\hline $\mathrm{HC} 10$ & 0.43 & $8.3 \pm 0.5$ & $58 \pm 4$ & $0.0120 \pm 0.0007$ & $31 \pm 2$ \\
\hline
\end{tabular}


145 M. A. Misdaq et al:: Radon, Thoron and Progeny Measured in Urban Health Centres and the Resulting Radiation Doses to Doctors, Nurses and Patients from the Inhalation of Air

\subsection{Committed Effective Doses Due to Short-Lived Alpha- Emitting ${ }^{222}$ Rn Decay Products in the Respiratory Tract of Doctors, Nurses and Patients}

Committed equivalent doses per hour of exposure due to the attached and unattached fractions of the short-lived alphaemitting ${ }^{222} \mathrm{Rn}$ daughters ${ }^{218} \mathrm{Po}\left(H_{T}^{a}\left({ }^{218} \mathrm{Po}\right)\right.$ and $\left.H_{T}^{u}\left({ }^{218} \mathrm{Po}\right)\right)$ and ${ }^{214} \mathrm{Po}\left(H_{T}^{a}\left({ }^{214} \mathrm{Po}\right)\right.$ and $\left.H_{T}^{u}\left({ }^{214} \mathrm{Po}\right)\right)$ were evaluated in the respiratory tract of doctors, nurses and patients from the inhalation of air in the studied health centres (Tables 4-8). The statistical relative uncertainty of the committed equivalent dose determination is about $10 \%$.

Table 4. Data obtained for committed equivalent doses due to the unattached (a) and attached (b) fractions of the ${ }^{218}$ Po and ${ }^{214}$ Po radon progeny in the tissues of the respiratory tract from the inhalation of air by male doctors in different health centres. Given errors correspond to one-sigma uncertainties.

Table $4(a)$

\begin{tabular}{|c|c|c|c|c|c|c|c|c|c|c|}
\hline \multirow{3}{*}{$\begin{array}{l}\text { Health } \\
\text { centre }\end{array}$} & \multicolumn{5}{|l|}{${ }^{218} \mathrm{Po}$} & \multicolumn{5}{|l|}{${ }^{214} \mathrm{Po}$} \\
\hline & \multicolumn{3}{|c|}{ Thoracic region } & \multicolumn{2}{|c|}{ Extrathoracic region } & \multicolumn{3}{|c|}{ Thoracic region } & \multicolumn{2}{|c|}{ Extrathoracic region } \\
\hline & $\begin{array}{l}H_{A I}^{u} \\
\left(\mathbf{x 1 0}{ }^{10} \mathbf{S v} \cdot \mathbf{y}^{-1}\right)\end{array}$ & $\begin{array}{l}H_{b b}^{u} \\
\left(\mathbf{x} 10^{7} \mathbf{S v} \cdot \mathbf{y}^{-1}\right)\end{array}$ & $\begin{array}{l}H_{B B}^{u} \\
\left(\mathbf{x 1 0}^{\mathbf{7}} \mathbf{S v} \cdot \mathbf{y}^{-1}\right)\end{array}$ & $\begin{array}{l}H_{E T 2}^{u} \\
\left(\mathbf{x} 10^{5} \mathbf{S v} \cdot \mathbf{y}^{-1}\right)\end{array}$ & $\begin{array}{l}H_{E T 1}^{u} \\
\left(\mathbf{x 1 0}^{\mathbf{7}} \mathbf{S v} \cdot \mathbf{y}^{-\mathbf{1}}\right)\end{array}$ & $\begin{array}{l}H_{A I}^{u} \\
\left(\mathbf{x 1 0}{ }^{19} \mathbf{S v} \cdot \mathbf{y}^{-1}\right)\end{array}$ & $\begin{array}{l}H_{b b}^{u} \\
\left(\mathbf{x 1 0} 0^{15} \mathbf{S v} \cdot \mathbf{y}^{-1}\right)\end{array}$ & $\begin{array}{l}H_{B B}^{u} \\
\left(\mathbf{x 1 0} 0^{15} \mathbf{S v} \cdot \mathbf{y}^{-1}\right)\end{array}$ & $\begin{array}{l}H_{E T 2}^{u} \\
\left(\mathbf{x 1 0}{ }^{13} \mathbf{S v} \cdot \mathbf{y}^{-1}\right)\end{array}$ & $\begin{array}{l}H_{E T 1}^{u} \\
\left(\mathbf{x 1 0} \mathbf{1}^{\mathbf{1 5}} \mathbf{S v} \cdot \mathbf{y}^{-\mathbf{1}}\right)\end{array}$ \\
\hline $\mathrm{HC} 1$ & $0.32 \pm 0.03$ & $4.8 \pm 0.4$ & $5.8 \pm 0.5$ & $2.0 \pm 0.2$ & $5.7 \pm 0.5$ & $0.46 \pm 0.04$ & $0.69 \pm 0.06$ & $0.85 \pm 0.07$ & $0.36 \pm 0.03$ & $0.82 \pm 0.07$ \\
\hline $\mathrm{HC} 2$ & $0.32 \pm 0.03$ & $4.8 \pm 0.4$ & $5.8 \pm 0.5$ & $2.0 \pm 0.2$ & $5.7 \pm 0.5$ & $0.46 \pm 0.04$ & $0.69 \pm 0.06$ & $0.85 \pm 0.07$ & $0.36 \pm 0.03$ & $0.82 \pm 0.07$ \\
\hline $\mathrm{HC} 3$ & $0.57 \pm 0.06$ & $8.5 \pm 0.8$ & $10 \pm 1$ & $3.5 \pm 0.3$ & $10 \pm 1$ & $0.83 \pm 0.07$ & $1.2 \pm 0.1$ & $1.5 \pm 0.1$ & $0.66 \pm 0.06$ & $1.5 \pm 0.1$ \\
\hline HC5 & $0.54 \pm 0.06$ & $8.1 \pm 0.8$ & $10 \pm 1$ & $3.4 \pm 0.3$ & $9.7 \pm 0.7$ & $0.79 \pm 0.07$ & $1.2 \pm 0.1$ & $1.4 \pm 0.1$ & $0.63 \pm 0.06$ & $1.4 \pm 0.1$ \\
\hline HC6 & $0.51 \pm 0.05$ & $7.7 \pm 0.7$ & $9.4 \pm 0.9$ & $3.2 \pm 0.3$ & $9.1 \pm 0.7$ & $0.75 \pm 0.07$ & $1.1 \pm 0.1$ & $1.4 \pm 0.1$ & $0.59 \pm 0.05$ & $1.3 \pm 0.1$ \\
\hline $\mathrm{HC} 7$ & $0.33 \pm 0.03$ & $5.0 \pm 0.5$ & $6.0 \pm 0.6$ & $2.1 \pm 0.2$ & $5.9 \pm 0.5$ & $0.48 \pm 0.04$ & $0.72 \pm 0.06$ & $0.90 \pm 0.07$ & $0.38 \pm 0.03$ & $0.85 \pm 0.07$ \\
\hline $\mathrm{HC} 8$ & $0.36 \pm 0.03$ & $5.4 \pm 0.4$ & $6.6 \pm 0.6$ & $2.2 \pm 0.2$ & $6.4 \pm 0.5$ & $0.52 \pm 0.04$ & $0.78 \pm 0.06$ & $0.9 \pm 0.07$ & $0.41 \pm 0.03$ & $0.93 \pm 0.07$ \\
\hline HC9 & $0.66 \pm 0.06$ & $10 \pm 1$ & $12 \pm 1$ & $4.1 \pm 0.4$ & $12 \pm 1$ & $0.97 \pm 0.07$ & $1.4 \pm 0.1$ & $1.8 \pm 0.1$ & $0.77 \pm 0.07$ & $1.7 \pm 0.1$ \\
\hline $\mathrm{HC} 10$ & $0.42 \pm 0.04$ & $6.4 \pm 0.6$ & $7.8 \pm 0.7$ & $2.6 \pm 0.2$ & $7.6 \pm 0.7$ & $0.62 \pm 0.04$ & $0.9 \pm 0.07$ & $1.1 \pm 0.1$ & $0.49 \pm 0.04$ & $1.1 \pm 0.1$ \\
\hline
\end{tabular}

Table $4(b)$

\begin{tabular}{|c|c|c|c|c|c|c|c|c|c|c|}
\hline \multirow{3}{*}{$\begin{array}{l}\text { Health } \\
\text { centre }\end{array}$} & \multicolumn{5}{|l|}{${ }^{218} \mathrm{Po}$} & \multicolumn{5}{|l|}{${ }^{214} \mathrm{Po}$} \\
\hline & \multicolumn{3}{|c|}{ Thoracic region } & \multicolumn{2}{|c|}{ Extrathoracic region } & \multicolumn{3}{|c|}{ Thoracic region } & \multicolumn{2}{|c|}{ Extrathoracic region } \\
\hline & $\begin{array}{l}H_{A I}^{a} \\
\left(\mathbf{x} 10^{9} \mathbf{S v}^{-1} \mathbf{y}^{-1}\right)\end{array}$ & $\begin{array}{l}H_{b b}^{a} \\
\left(\mathbf{x} 10^{7} \mathbf{S v}^{-1} \mathbf{y}^{-1}\right)\end{array}$ &  & $\begin{array}{l}H_{E T 2}^{a} \\
\left(\mathbf{x 1 0 ^ { 5 }} \mathbf{S v}^{-1} \mathbf{y}^{-1}\right)\end{array}$ & $\begin{array}{l}H_{E T 1}^{a} \\
\left(\mathbf{x 1 0 ^ { 7 }} \mathbf{S v}^{-1} \mathbf{y}^{-1}\right)\end{array}$ & $\begin{array}{l}H_{A I}^{a} \\
\left(\mathbf{x 1 0} 0^{15} \mathbf{S v} \cdot \mathbf{y}^{-1}\right)\end{array}$ & $\begin{array}{l}H_{b b}^{a} \\
\left(\mathbf{x 1 0} 0^{13} \mathbf{S v} \cdot \mathbf{y}^{-1}\right)\end{array}$ & $\begin{array}{l}H_{B B}^{a} \\
\left(\mathbf{x 1 0} 0^{13} \mathbf{S v} \cdot \mathbf{y}^{-1}\right)\end{array}$ & $\begin{array}{l}H_{E T 2}^{a} \\
\left(\mathbf{x 1 0} \mathbf{1}^{11} \mathbf{S v} \cdot \mathbf{y}^{-1}\right)\end{array}$ & $\begin{array}{l}H_{E T 1}^{a} \\
\left(\mathbf{x 1 0 ^ { 1 3 }} \mathbf{S v} \cdot \mathbf{y}^{-1}\right)\end{array}$ \\
\hline $\mathrm{HC} 1$ & $9.1 \pm 0.8$ & $10 \pm 1$ & $3.4 \pm 0.3$ & $2.1 \pm 0.2$ & $5.4 \pm 0.5$ & $4.5 \pm 0.4$ & $5.2 \pm 0.5$ & $1.7 \pm 0.1$ & $1.4 \pm 0.1$ & $2.7 \pm 0.2$ \\
\hline $\mathrm{HC} 2$ & $9.1 \pm 0.8$ & $10 \pm 1$ & $3.4 \pm 0.3$ & $2.1 \pm 0.2$ & $5.4 \pm 0.5$ & $4.5 \pm 0.4$ & $5.2 \pm 0.5$ & $1.7 \pm 0.1$ & $1.4 \pm 0.1$ & $2.7 \pm 0.2$ \\
\hline $\mathrm{HC} 3$ & $16 \pm 1$ & $19 \pm 1$ & $6.1 \pm 0.6$ & $3.8 \pm 0.3$ & $9.8 \pm 0.9$ & $9.5 \pm 0.8$ & $11 \pm 1$ & $3.6 \pm 0.3$ & $2.9 \pm 0.2$ & $5.7 \pm 0.5$ \\
\hline $\mathrm{HC} 4$ & $20 \pm 2$ & $23 \pm 2$ & $7.7 \pm 0.7$ & $4.8 \pm 0.4$ & $12 \pm 1$ & $12 \pm 1$ & $14 \pm 1$ & $4.6 \pm 0.4$ & $3.7 \pm 0.3$ & $7.5 \pm 0.6$ \\
\hline HC5 & $15 \pm 1$ & $18 \pm 1$ & $5.8 \pm 0.5$ & $3.6 \pm 0.3$ & $9.3 \pm 0.8$ & $9.0 \pm 0.8$ & $10 \pm 1$ & $3.4 \pm 0.3$ & $2.7 \pm 0.2$ & $5.4 \pm 0.4$ \\
\hline HC6 & $15 \pm 1$ & $17 \pm 1$ & $5.5 \pm 0.5$ & $3.4 \pm 0.3$ & $8.8 \pm 0.8$ & $8.5 \pm 0.8$ & $10 \pm 1$ & $3.2 \pm 0.3$ & $2.5 \pm 0.2$ & $5.1 \pm 0.4$ \\
\hline $\mathrm{HC} 7$ & $9.4 \pm 0.8$ & $11 \pm 1$ & $3.5 \pm 0.3$ & $2.2 \pm 0.2$ & $5.6 \pm 0.5$ & $4.8 \pm 0.4$ & $5.5 \pm 0.5$ & $1.8 \pm 0.1$ & $1.4 \pm 0.1$ & $2.9 \pm 0.2$ \\
\hline HC8 & $10 \pm 1$ & $12 \pm 1$ & $3.9 \pm 0.3$ & $2.4 \pm 0.2$ & $6.2 \pm 0.5$ & $5.5 \pm 0.5$ & $6.3 \pm 0.5$ & $2.1 \pm 0.2$ & $1.6 \pm 0.2$ & $3.3 \pm 0.3$ \\
\hline HC9 & $19 \pm 1$ & $22 \pm 2$ & $7.1 \pm 0.7$ & $4.4 \pm 0.4$ & $11 \pm 1$ & $11 \pm 1$ & $13 \pm 1$ & $4.3 \pm 0.4$ & $3.4 \pm 0.3$ & $6.8 \pm 0.6$ \\
\hline $\mathrm{HC} 10$ & $12 \pm 1$ & $14 \pm 1$ & $4.6 \pm 0.4$ & $2.8 \pm 0.2$ & $7.3 \pm 0.7$ & $6.7 \pm 0.5$ & $8.0 \pm 0.7$ & $2.5 \pm 0.2$ & $2.0 \pm 0.2$ & $4.1 \pm 0.4$ \\
\hline
\end{tabular}

Table 5. Data obtained for committed equivalent doses due to the unattached (a) and attached (b) fractions of the ${ }^{218}$ Po and ${ }^{214} P o$ radon progeny in the tissues of the respiratory tract from the inhalation of air by female nurses in different health centers. Given errors correspond to one-sigma uncertainties.

Table 5(a)

\begin{tabular}{|c|c|c|c|c|c|c|c|c|c|c|}
\hline \multirow{3}{*}{$\begin{array}{l}\text { Health } \\
\text { centre }\end{array}$} & \multicolumn{5}{|l|}{${ }^{218} \mathrm{Po}$} & \multicolumn{5}{|l|}{${ }^{214} \mathrm{Po}$} \\
\hline & \multicolumn{3}{|c|}{ Thoracic region } & \multicolumn{2}{|c|}{ Extrathoracic region } & \multicolumn{3}{|c|}{ Thoracic region } & \multicolumn{2}{|c|}{ Extrathoracic region } \\
\hline & $\begin{array}{l}H_{A I}^{u} \\
\left(\mathbf{x 1 0}{ }^{10} \mathbf{S v} \cdot \mathbf{y}^{-1}\right)\end{array}$ & $\begin{array}{l}H_{b b}^{u} \\
\left(\mathbf{x 1 0}^{7} \mathbf{S v} \cdot \mathbf{y}^{-1}\right)\end{array}$ & $\begin{array}{l}H_{B B}^{u} \\
\left(\mathbf{x 1 0}^{7} \mathbf{S v} \cdot \mathbf{y}^{-1}\right)\end{array}$ & $\begin{array}{l}H_{E T 2}^{u} \\
\left(\mathbf{x} 10^{5} \mathbf{S v} \cdot \mathbf{y}^{-1}\right)\end{array}$ & $\begin{array}{l}H_{E T 1}^{u} \\
\left(\mathbf{x 1 0} \mathbf{S}^{7} \mathbf{S v} \cdot \mathbf{y}^{-1}\right)\end{array}$ & $\begin{array}{l}H_{A I}^{u} \\
\left(\mathbf{x 1 0}{ }^{19} \mathbf{S v} \cdot \mathbf{y}^{-1}\right)\end{array}$ & $\begin{array}{l}H_{b b}^{u} \\
\left(\mathbf{x 1 0}{ }^{15} \mathbf{S v} \cdot \mathbf{y}^{-1}\right)\end{array}$ & $\begin{array}{l}H_{B B}^{u} \\
\left(\mathbf{x 1 0} 0^{15} \mathbf{S v} \cdot \mathbf{y}^{-1}\right)\end{array}$ & $\begin{array}{l}H_{E T 2}^{u} \\
\left(\mathbf{x} 10^{13} \mathbf{S v} \cdot \mathbf{y}^{-1}\right)\end{array}$ & $\begin{array}{l}H_{E T 1}^{u} \\
\left(\mathbf{x} 10^{15} \mathbf{S v} \cdot \mathbf{y}^{-1}\right)\end{array}$ \\
\hline $\mathrm{HC} 1$ & $0.42 \pm 0.04$ & $5.1 \pm 0.4$ & $6.8 \pm 0.6$ & $2.4 \pm 0.2$ & $7.1 \pm 0.7$ & $0.60 \pm 0.05$ & $0.70 \pm 0.05$ & $1.0 \pm 0.1$ & $0.40 \pm 0.03$ & $1.0 \pm 0.1$ \\
\hline $\mathrm{HC} 2$ & $0.41 \pm 0.04$ & $5.0 \pm 0.4$ & $6.8 \pm 0.6$ & $2.4 \pm 0.2$ & $7.0 \pm 0.7$ & $0.60 \pm 0.05$ & $0.70 \pm 0.05$ & $1.0 \pm 0.1$ & $0.40 \pm 0.03$ & $1.0 \pm 0.1$ \\
\hline $\mathrm{HC} 3$ & $0.73 \pm 0.06$ & $8.9 \pm 0.8$ & $12 \pm 1$ & $4.3 \pm 0.4$ & $12 \pm 1$ & $1.1 \pm 0.1$ & $1.3 \pm 0.1$ & $1.8 \pm 0.2$ & $0.80 \pm 0.07$ & $1.8 \pm 0.2$ \\
\hline $\mathrm{HC} 4$ & $0.46 \pm 0.04$ & $5.7 \pm 0.5$ & $7.6 \pm 0.7$ & $2.7 \pm 0.2$ & $7.9 \pm 0.7$ & $0.70 \pm 0.05$ & $0.80 \pm 0.05$ & $1.1 \pm 0.1$ & $0.50 \pm 0.04$ & $1.2 \pm 0.1$ \\
\hline HC6 & $0.55 \pm 0.05$ & $6.8 \pm 0.6$ & $9.1 \pm 0.8$ & $3.2 \pm 0.3$ & $9.5 \pm 0.8$ & $0.80 \pm 0.05$ & $1.0 \pm 0.1$ & $1.3 \pm 0.1$ & $0.60 \pm 0.05$ & $1.4 \pm 0.1$ \\
\hline $\mathrm{HC7}$ & $0.42 \pm 0.04$ & $5.2 \pm 0.4$ & $6.9 \pm 0.6$ & $2.5 \pm 0.2$ & $7.2 \pm 0.7$ & $0.60 \pm 0.05$ & $0.70 \pm 0.05$ & $1.0 \pm 0.1$ & $0.50 \pm 0.04$ & $1.0 \pm 0.1$ \\
\hline $\mathrm{HC} 8$ & $0.41 \pm 0.04$ & $5.1 \pm 0.4$ & $6.8 \pm 0.6$ & $2.4 \pm 0.2$ & $7.1 \pm 0.7$ & $0.60 \pm 0.05$ & $0.70 \pm 0.05$ & $1.0 \pm 0.1$ & $0.40 \pm 0.03$ & $1.0 \pm 0.1$ \\
\hline HC9 & $0.74 \pm 0.06$ & $9.1 \pm 0.8$ & $12 \pm 1$ & $4.3 \pm 0.4$ & $13 \pm 1$ & $1.1 \pm 0.1$ & $1.3 \pm 0.1$ & $1.8 \pm 0.2$ & $0.80 \pm 0.07$ & $1.9 \pm 0.1$ \\
\hline $\mathrm{HC} 10$ & $0.47 \pm 0.04$ & $5.7 \pm 0.5$ & $7.7 \pm 0.7$ & $2.7 \pm 0.2$ & $7.9 \pm 0.7$ & $0.70 \pm 0.05$ & $0.80 \pm 0.05$ & $1.1 \pm 0.1$ & $0.50 \pm 0.04$ & $1.2 \pm 0.1$ \\
\hline
\end{tabular}


Table $5(b)$

\begin{tabular}{|c|c|c|c|c|c|c|c|c|c|c|}
\hline \multirow{3}{*}{$\begin{array}{l}\text { Health } \\
\text { centre }\end{array}$} & \multicolumn{5}{|l|}{${ }^{218} \mathrm{Po}$} & \multicolumn{5}{|l|}{${ }^{214} \mathbf{P o}$} \\
\hline & \multicolumn{3}{|c|}{ Thoracic region } & \multicolumn{2}{|c|}{ Extrathoracic region } & \multicolumn{2}{|c|}{ Thoracic region } & \multicolumn{3}{|c|}{ Extrathoracic region } \\
\hline & $\begin{array}{l}H_{A I}^{a} \\
\left(\mathbf{x 1 0} 0^{9} \mathbf{S v} \cdot \mathbf{y}^{-1}\right)\end{array}$ & $\begin{array}{l}H_{b b}^{a} \\
\left(\mathbf{x 1 0 ^ { 7 }} \mathbf{S v} \cdot \mathbf{y}^{-1}\right)\end{array}$ & $\begin{array}{l}H_{B B}^{a} \\
\left(\mathbf{x 1 0} \mathbf{S v}^{7} \cdot \mathbf{y}^{-1}\right)\end{array}$ & $\begin{array}{l}H_{E T 2}^{a} \\
\left(\mathbf{x 1 0} \mathbf{S v}^{-1} \mathbf{y}^{-1}\right)\end{array}$ & $\begin{array}{l}H_{E T 1}^{a} \\
\left(\mathbf{x 1 0 ^ { 7 }} \mathbf{S v} \cdot \mathbf{y}^{-1}\right)\end{array}$ & $\begin{array}{l}H_{A I}^{a} \\
\left(\mathbf{x 1 0}{ }^{15} \mathbf{S v} \cdot \mathbf{y}^{-1}\right) \\
\end{array}$ & $\begin{array}{l}H_{b b}^{a} \\
\left(\mathbf{x 1 0} 0^{13} \mathbf{S v} \cdot \mathbf{y}^{-1}\right) \\
\end{array}$ & $\begin{array}{l}H_{B B}^{a} \\
\left(\mathbf{x 1 0 ^ { 1 3 }} \mathbf{S v} \cdot \mathbf{y}^{-1}\right)\end{array}$ & $\begin{array}{l}H_{E T 2}^{a} \\
\left(\mathbf{x 1 0 ^ { 1 1 }} \mathbf{S v}^{-1} \mathbf{y}^{-1}\right) \\
\end{array}$ & $\begin{array}{l}H_{E T 1}^{a} \\
\left(\mathbf{x 1 0 ^ { 1 3 }} \mathbf{S v} \cdot \mathbf{y}^{-1}\right)\end{array}$ \\
\hline $\mathrm{HCl}$ & $12 \pm 1$ & $11 \pm 1$ & $4.0 \pm 0.3$ & $2.6 \pm 0.2$ & $6.8 \pm 0.6$ & $6.1 \pm 0.6$ & $5.8 \pm 0.5$ & $2.1 \pm 0.2$ & $1.7 \pm 0.1$ & $3.6 \pm 0.3$ \\
\hline $\mathrm{HC} 2$ & $11 \pm 1$ & $11 \pm 1$ & $3.9 \pm 0.3$ & $2.6 \pm 0.2$ & $6.7 \pm 0.6$ & $6.0 \pm 0.6$ & $5.7 \pm 0.5$ & $2.0 \pm 0.2$ & $1.7 \pm 0.1$ & $3.5 \pm 0.3$ \\
\hline $\mathrm{HC} 3$ & $21 \pm 2$ & $20 \pm 2$ & $7.1 \pm 0.6$ & $4.6 \pm 0.4$ & $12 \pm 1$ & $12 \pm 1$ & $12 \pm 1$ & $4.2 \pm 0.4$ & $3.5 \pm 0.3$ & $7.2 \pm 0.6$ \\
\hline $\mathrm{HC} 4$ & $13 \pm 1$ & $12 \pm 1$ & $4.5 \pm 0.3$ & $2.9 \pm 0.2$ & $7.7 \pm 0.7$ & $7.1 \pm 0.7$ & $6.7 \pm 0.6$ & $2.4 \pm 0.2$ & $2.0 \pm 0.2$ & $4.1 \pm 0.4$ \\
\hline HC5 & $13 \pm 1$ & $13 \pm 1$ & $4.6 \pm 0.4$ & $3.0 \pm 0.2$ & $7.9 \pm 0.7$ & $7.4 \pm 0.7$ & $7.0 \pm 0.6$ & $2.5 \pm 0.2$ & $2.1 \pm 0.2$ & $4.3 \pm 0.4$ \\
\hline HC6 & $16 \pm 1$ & $15 \pm 1$ & $5.4 \pm 0.4$ & $3.5 \pm 0.2$ & $9.2 \pm 0.9$ & $8.9 \pm 0.8$ & $8.4 \pm 0.7$ & $3.0 \pm 0.3$ & $2.5 \pm 0.2$ & $5.2 \pm 0.5$ \\
\hline $\mathrm{HC} 7$ & $12 \pm 1$ & $11 \pm 1$ & $4.1 \pm 0.3$ & $2.6 \pm 0.2$ & $6.9 \pm 0.6$ & $6.2 \pm 0.6$ & $5.9 \pm 0.5$ & $2.1 \pm 0.2$ & $1.8 \pm 0.1$ & $3.6 \pm 0.3$ \\
\hline $\mathrm{HC} 8$ & $12 \pm 1$ & $11 \pm 1$ & $4.0 \pm 0.3$ & $2.6 \pm 0.2$ & $6.8 \pm 0.6$ & $6.0 \pm 0.6$ & $5.7 \pm 0.5$ & $2.1 \pm 0.2$ & $1.7 \pm 0.1$ & $3.5 \pm 0.3$ \\
\hline HC9 & $21 \pm 2$ & $20 \pm 2$ & $7.2 \pm 0.6$ & $4.7 \pm 0.4$ & $12 \pm 1$ & $13 \pm 1$ & $12 \pm 1$ & $4.3 \pm 0.4$ & $3.5 \pm 0.3$ & $7.3 \pm 0.6$ \\
\hline $\mathrm{HC} 10$ & $13 \pm 1$ & $12 \pm 1$ & $4.5 \pm 0.3$ & $2.9 \pm 0.2$ & $7.7 \pm 0.7$ & $7.2 \pm 0.7$ & $6.8 \pm 0.6$ & $2.4 \pm 0.2$ & $2.0 \pm 0.2$ & $4.2 \pm 0.4$ \\
\hline
\end{tabular}

Table 6. Data obtained for committed equivalent doses due to the unattached (a) and attached (b) fractions of the ${ }^{218}$ Po and ${ }^{214}$ Po radon progeny in the tissues of the respiratory tract from the inhalation of air by 15 y girls in different health centres. Given errors correspond to one-sigma uncertainties.

Table $6(a)$

\begin{tabular}{|c|c|c|c|c|c|c|c|c|c|c|}
\hline \multirow{3}{*}{$\begin{array}{l}\text { Health } \\
\text { centre }\end{array}$} & \multicolumn{5}{|l|}{${ }^{218} \mathrm{Po}$} & \multicolumn{5}{|l|}{${ }^{214} \mathrm{Po}$} \\
\hline & \multicolumn{3}{|c|}{ Thoracic region } & \multicolumn{2}{|c|}{ Extrathoracic region } & \multicolumn{3}{|c|}{ Thoracic region } & \multicolumn{2}{|c|}{ Extrathoracic region } \\
\hline & $\begin{array}{l}H_{A I}^{u} \\
\left(\mathbf{x 1 0}^{12} \mathbf{S v}_{\left.\cdot \mathbf{y}^{-1}\right)}\right)\end{array}$ & 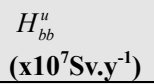 & $\begin{array}{l}H_{B B}^{u} \\
\left(\mathbf{x 1 0}^{7} \mathbf{S v} \cdot \mathbf{y}^{-1}\right)\end{array}$ & 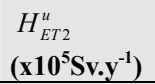 & $\begin{array}{l}H_{E T 1}^{u} \\
\left(\mathbf{x 1 0}^{\mathbf{7}} \mathbf{S v} \cdot \mathbf{y}^{-1}\right)\end{array}$ & $\begin{array}{l}H_{A I}^{u} \\
\left(\mathbf{x 1 0 ^ { 2 1 }} \mathbf{S v}^{-1} \mathbf{y}^{-1}\right)\end{array}$ & $\begin{array}{l}H_{b b}^{u} \\
\left(\mathbf{x 1 0}{ }^{15} \mathbf{S v}^{-1} \mathbf{y}^{-1}\right)\end{array}$ & $\begin{array}{l}H_{B B}^{u} \\
\left(\mathbf{x 1 0 ^ { 1 5 }} \mathbf{S v}^{-1} \mathbf{y}^{-1}\right.\end{array}$ & 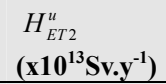 & 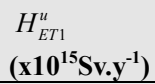 \\
\hline $\mathrm{HC} 1$ & $0.39 \pm 0.03$ & $0.80 \pm 0.07$ & $3.0 \pm 0.3$ & $0.90 \pm 0.07$ & $2.8 \pm 0.2$ & $0.56 \pm 0.04$ & $0.12 \pm 0.01$ & $0.45 \pm 0.03$ & $0.18 \pm 0.01$ & $0.40 \pm 0.04$ \\
\hline $\mathrm{HC} 2$ & $0.35 \pm 0.03$ & $0.70 \pm 0.06$ & $2.7 \pm 0.2$ & $0.80 \pm 0.07$ & $2.5 \pm 0.2$ & $0.50 \pm 0.04$ & $0.11 \pm 0.01$ & $0.40 \pm 0.03$ & $0.16 \pm 0.01$ & $0.36 \pm 0.03$ \\
\hline $\mathrm{HC} 3$ & $0.56 \pm 0.05$ & $1.2 \pm 0.1$ & $4.4 \pm 0.3$ & $1.4 \pm 0.1$ & $4.0 \pm 0.3$ & $0.82 \pm 0.07$ & $0.18 \pm 0.01$ & $0.65 \pm 0.05$ & $0.26 \pm 0.02$ & $0.59 \pm 0.05$ \\
\hline $\mathrm{HC} 4$ & $0.34 \pm 0.03$ & $0.70 \pm 0.06$ & $2.6 \pm 0.2$ & $0.80 \pm 0.07$ & $2.4 \pm 0.2$ & $0.48 \pm 0.04$ & $0.10 \pm 0.01$ & $0.38 \pm 0.03$ & $0.15 \pm 0.01$ & $0.35 \pm 0.03$ \\
\hline HC5 & $0.34 \pm 0.03$ & $0.70 \pm 0.06$ & $2.6 \pm 0.2$ & $0.80 \pm 0.07$ & $2.4 \pm 0.2$ & $0.48 \pm 0.04$ & $0.10 \pm 0.01$ & $0.38 \pm 0.03$ & $0.15 \pm 0.01$ & $0.35 \pm 0.03$ \\
\hline HC6 & $0.42 \pm 0.04$ & $0.90 \pm 0.07$ & $3.3 \pm 0.3$ & $1.0 \pm 0.1$ & $3.0 \pm 0.3$ & $0.61 \pm 0.05$ & $0.13 \pm 0.01$ & $0.48 \pm 0.04$ & $0.19 \pm 0.01$ & $0.44 \pm 0.03$ \\
\hline $\mathrm{HC} 7$ & $0.31 \pm 0.03$ & $0.70 \pm 0.06$ & $2.5 \pm 0.2$ & $0.80 \pm 0.07$ & $2.2 \pm 0.2$ & $0.45 \pm 0.04$ & $0.10 \pm 0.01$ & $0.36 \pm 0.03$ & $0.14 \pm 0.01$ & $0.32 \pm 0.02$ \\
\hline $\mathrm{HC} 8$ & $0.32 \pm 0.03$ & $0.70 \pm 0.06$ & $2.5 \pm 0.2$ & $0.80 \pm 0.07$ & $2.3 \pm 0.2$ & $0.46 \pm 0.04$ & $0.10 \pm 0.01$ & $0.37 \pm 0.03$ & $0.14 \pm 0.01$ & $0.33 \pm 0.02$ \\
\hline HC9 & $0.57 \pm 0.05$ & $1.2 \pm 0.1$ & $4.4 \pm 0.3$ & $1.4 \pm 0.1$ & $4.1 \pm 0.3$ & $0.82 \pm 0.07$ & $0.18 \pm 0.02$ & $0.66 \pm 0.05$ & $0.26 \pm 0.02$ & $0.59 \pm 0.05$ \\
\hline $\mathrm{HC} 10$ & $0.36 \pm 0.03$ & $0.80 \pm 0.07$ & $2.8 \pm 0.2$ & $0.90 \pm 0.07$ & $2.6 \pm 0.2$ & $0.52 \pm 0.04$ & $0.11 \pm 0.01$ & $0.41 \pm 0.03$ & $0.16 \pm 0.01$ & $0.37 \pm 0.03$ \\
\hline
\end{tabular}

Table 6 (b)

\begin{tabular}{|c|c|c|c|c|c|c|c|c|c|c|}
\hline \multirow{3}{*}{$\begin{array}{l}\text { Health } \\
\text { centre }\end{array}$} & \multicolumn{5}{|l|}{${ }^{218} \mathrm{Po}$} & \multicolumn{5}{|l|}{${ }^{214} \mathrm{Po}$} \\
\hline & \multicolumn{3}{|c|}{ Thoracic region } & \multicolumn{2}{|c|}{ Extrathoracic region } & \multicolumn{3}{|c|}{ Thoracic region } & \multicolumn{2}{|c|}{ Extrathoracic region } \\
\hline & 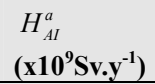 & $\begin{array}{l}H_{b b}^{a} \\
\left(\mathbf{x 1 0}^{7} \mathbf{S v}^{\left.-\mathbf{y}^{-1}\right)}\right.\end{array}$ & 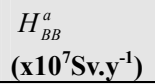 & $\begin{array}{l}H_{E T 2}^{a} \\
\left(\mathbf{x 1 0 ^ { 5 }} \text { Sv. }^{-1}\right)\end{array}$ & $\begin{array}{l}H_{E T 1}^{a} \\
\left(\mathbf{x 1 0 ^ { 7 }} \mathbf{S v} \cdot \mathbf{y}^{-1}\right)\end{array}$ & $\begin{array}{l}H_{A I}^{a} \\
\left(\mathbf{x 1 0}^{16} \mathbf{S v} \cdot \mathbf{y}^{-1}\right)\end{array}$ & $\begin{array}{l}H_{b b}^{a} \\
\left(\mathbf{x 1 0}{ }^{13} \mathbf{S v}^{-\mathbf{y}^{-1}}\right)\end{array}$ & $\begin{array}{l}H_{B B}^{a} \\
\left(\mathbf{x 1 0}{ }^{13} \text { Sv. }^{-1}\right)\end{array}$ & 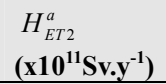 & $\begin{array}{l}H_{E T 1}^{a} \\
\left(\mathbf{x 1 0 ^ { 1 3 }} \mathbf{S v . y}^{-1}\right)\end{array}$ \\
\hline $\mathrm{HC} 1$ & $3.8 \pm 0.3$ & $6.1 \pm 0.6$ & $1.9 \pm 0.1$ & $0.50 \pm 0.04$ & $1.5 \pm 0.1$ & $20 \pm 2$ & $3.3 \pm 0.3$ & $5.2 \pm 0.5$ & $0.40 \pm 0.04$ & $0.80 \pm 0.07$ \\
\hline $\mathrm{HC} 2$ & $3.4 \pm 0.3$ & $5.4 \pm 0.5$ & $1.7 \pm 0.1$ & $0.50 \pm 0.04$ & $1.3 \pm 0.1$ & $17 \pm 1$ & $2.8 \pm 0.2$ & $4.4 \pm 0.4$ & $0.30 \pm 0.03$ & $0.70 \pm 0.06$ \\
\hline $\mathrm{HC} 3$ & $5.6 \pm 0.5$ & $8.9 \pm 0.8$ & $2.8 \pm 0.2$ & $0.80 \pm 0.06$ & $2.2 \pm 0.2$ & $32 \pm 3$ & $5.2 \pm 0.4$ & $8.3 \pm 0.8$ & $0.60 \pm 0.05$ & $1.3 \pm 0$, \\
\hline $\mathrm{HC} 4$ & $3.3 \pm 0.3$ & $5.3 \pm 0.5$ & $1.7 \pm 0.1$ & $0.50 \pm 0.04$ & $1.3 \pm 0.1$ & $17 \pm 1$ & $2.7 \pm 0.2$ & $4.2 \pm 0.4$ & $0.30 \pm 0.03$ & $0.70 \pm 006$ \\
\hline HC5 & $3.3 \pm 0.3$ & $5.3 \pm 0.5$ & $1.7 \pm 0.1$ & $0.50 \pm 0.04$ & $1.3 \pm 0.1$ & $17 \pm 1$ & $2.7 \pm 0.2$ & $4.2 \pm 0.4$ & $0.30 \pm 0.03$ & $0.70 \pm 0.06$ \\
\hline HC6 & $4.1 \pm 0.4$ & $6.6 \pm 0.5$ & $2.1 \pm 0.2$ & $0.60 \pm 0.05$ & $1.6 \pm 0.1$ & $23 \pm 2$ & $3.6 \pm 0.3$ & $5.8 \pm 0.5$ & $0.40 \pm 0.04$ & $0.90 \pm 0.08$ \\
\hline $\mathrm{HC7}$ & $3.1 \pm 0.3$ & $4.9 \pm 0.4$ & $1.6 \pm 0.1$ & $0.40 \pm 0.03$ & $1.2 \pm 0.1$ & $14 \pm 1$ & $2.3 \pm 0.2$ & $3.7 \pm 0.3$ & $0.30 \pm 0.03$ & $0.60 \pm 0.05$ \\
\hline $\mathrm{HC} 8$ & $3.1 \pm 0.3$ & $5.0 \pm 0.4$ & $1.6 \pm 0.1$ & $0.40 \pm 0.03$ & $1.2 \pm 0.1$ & $15 \pm 1$ & $2.5 \pm 0.2$ & $3.9 \pm 0.3$ & $0.30 \pm 0.03$ & $0.60 \pm 0.05$ \\
\hline HC9 & $5.6 \pm 0.5$ & $9.0 \pm 0.8$ & $2.9 \pm 0.2$ & $0.80 \pm 0.06$ & $2.2 \pm 0.2$ & $32 \pm 3$ & $5.2 \pm 0.4$ & $8.3 \pm 0.8$ & $0.60 \pm 0.05$ & $1.3 \pm 0.1$ \\
\hline $\mathrm{HC} 10$ & $3.5 \pm 0.3$ & $5.6 \pm 0.5$ & $1.8 \pm 0.1$ & $0.50 \pm 0.04$ & $1.4 \pm 0.1$ & $18 \pm 1$ & $3.0 \pm 0.3$ & $4.7 \pm 0.4$ & $0.30 \pm 0.03$ & $0.70 \pm 0.06$ \\
\hline
\end{tabular}


147 M. A. Misdaq et al:: Radon, Thoron and Progeny Measured in Urban Health Centres and the Resulting Radiation Doses to Doctors, Nurses and Patients from the Inhalation of Air

Table 7. Data obtained for committed equivalent doses due to the unattached (a) and attached (b) fractions of the ${ }^{218}$ Po and ${ }^{214}$ Po radon progeny in the tissues of the respiratory tract from the inhalation of air by $10 y$ child in different health centres. Given errors correspond to one-sigma uncertainties.

Table 7 (a)

\begin{tabular}{|c|c|c|c|c|c|c|c|c|c|c|}
\hline \multirow{3}{*}{$\begin{array}{l}\text { Health } \\
\text { centre }\end{array}$} & \multicolumn{5}{|l|}{${ }^{218} \mathrm{Po}$} & \multicolumn{5}{|l|}{${ }^{214} \mathrm{Po}$} \\
\hline & \multicolumn{3}{|c|}{ Thoracic region } & \multicolumn{2}{|c|}{ Extrathoracic region } & \multicolumn{3}{|c|}{ Thoracic region } & \multicolumn{2}{|c|}{ Extrathoracic region } \\
\hline &  & $\begin{array}{l}H_{b b}^{u} \\
\left(\mathbf{x 1 0}^{7} \mathbf{S v}_{\mathbf{y}} \mathbf{y}^{-1}\right)\end{array}$ & $\begin{array}{l}H_{B B}^{u} \\
\left(\mathbf{x 1 0}^{7} \mathbf{S v}_{\mathbf{y}} \mathbf{y}^{-1}\right)\end{array}$ & 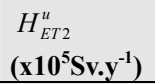 & $\begin{array}{l}H_{E T 1}^{u} \\
\left(\mathbf{x 1 0}^{7} \mathbf{S v} \cdot \mathbf{y}^{-1}\right)\end{array}$ & $\begin{array}{l}H_{A I}^{u} \\
\left(\mathbf{x 1 0}{ }^{21} \mathbf{S v} \cdot \mathbf{y}^{-1}\right)\end{array}$ & $\begin{array}{l}H_{b b}^{u} \\
\left(\mathbf{x 1 0}{ }^{15} \mathbf{S v}^{\left.-\mathbf{y}^{-1}\right)}\right.\end{array}$ & $\begin{array}{l}H_{B B}^{u} \\
\left(\mathbf{x 1 0 ^ { 1 5 }} \mathbf{S v}^{-1} \mathbf{y}^{-1}\right.\end{array}$ & $\begin{array}{l}H_{E T 2}^{u} \\
\left(\mathbf{x 1 0 ^ { 1 3 }} \mathbf{S v} \cdot \mathbf{y}^{-1}\right)\end{array}$ & $\begin{array}{l}H_{E T 1}^{u} \\
\left(\mathbf{x 1 0}{ }^{15} \mathbf{S v} \cdot \mathbf{y}^{-1}\right)\end{array}$ \\
\hline $\mathrm{HCl}$ & $0.90 \pm 0.08$ & $1.2 \pm 0.1$ & $3.7 \pm 0.3$ & $1.3 \pm 0.1$ & $3.4 \pm 0.3$ & $1.3 \pm 0.1$ & $0.20 \pm 0.01$ & $0.50 \pm 0.05$ & $0.20 \pm 0.02$ & $0.50 \pm 0.05$ \\
\hline $\mathrm{HC} 2$ & $0.80 \pm 0.07$ & $1.1 \pm 0.1$ & $3.3 \pm 0.3$ & $1.1 \pm 0.1$ & $3.1 \pm 0.3$ & $1.2 \pm 0.1$ & $0.16 \pm 0.01$ & $0.48 \pm 0.04$ & $0.21 \pm 0.02$ & $0.44 \pm 0.04$ \\
\hline $\mathrm{HC} 3$ & $1.3 \pm 0.1$ & $1.8 \pm 0.1$ & $5.4 \pm 0.5$ & $1.8 \pm 0.1$ & $5.0 \pm 0.5$ & $2.0 \pm 0.2$ & $0.27 \pm 0.02$ & $0.80 \pm 0.08$ & $0.34 \pm 0.03$ & $0.74 \pm 0.07$ \\
\hline $\mathrm{HC} 4$ & $0.80 \pm 0.07$ & $1.1 \pm 0.1$ & $3.2 \pm 0.3$ & $1.1 \pm 0.1$ & $3.0 \pm 0.3$ & $1.2 \pm 0.1$ & $0.16 \pm 0.01$ & $0.47 \pm 0.04$ & $0.20 \pm 0.02$ & $0.43 \pm 0.04$ \\
\hline HC5 & $0.80 \pm 0.07$ & $1.1 \pm 0.1$ & $3.2 \pm 0.3$ & $1.1 \pm 0.1$ & $3.0 \pm 0.3$ & $1.2 \pm 0.1$ & $0.16 \pm 0.01$ & $0.47 \pm 0.04$ & $0.20 \pm 0.02$ & $0.43 \pm 0.04$ \\
\hline HC6 & $1.0 \pm 0.1$ & $1.4 \pm 0.1$ & $4.0 \pm 0.4$ & $1.4 \pm 0.1$ & $3.7 \pm 0.3$ & $1.5 \pm 0.1$ & $0.20 \pm 0.02$ & $0.59 \pm 0.05$ & $0.25 \pm 0.02$ & $0.54 \pm 0.05$ \\
\hline $\mathrm{HC7}$ & $0.70 \pm 0.06$ & $1.0 \pm 0.1$ & $3.0 \pm 0.3$ & $1.0 \pm 0.1$ & $2.8 \pm 0.2$ & $1.1 \pm 0.1$ & $0.15 \pm 0.01$ & $0.44 \pm 0.04$ & $0.19 \pm 0.01$ & $0.40 \pm 0.04$ \\
\hline HC 8 & $0.70 \pm 0.06$ & $1.0 \pm 0.1$ & $3.1 \pm 0.3$ & $1.0 \pm 0.1$ & $2.9 \pm 0.2$ & $1.1 \pm 0.1$ & $0.15 \pm 0.01$ & $0.45 \pm 0.04$ & $0.19 \pm 0.01$ & $0.41 \pm 0.04$ \\
\hline HC9 & $1.3 \pm 0.1$ & $1.8 \pm 0.1$ & $5.4 \pm 0.5$ & $1.8 \pm 0.1$ & $5.0 \pm 0.5$ & $2.0 \pm 0.2$ & $0.27 \pm 0.02$ & $0.80 \pm 0.08$ & $0.34 \pm 0.03$ & $0.74 \pm 0.07$ \\
\hline $\mathrm{HC} 10$ & $0.80 \pm 0.07$ & $1.2 \pm 0.1$ & $3.4 \pm 0.3$ & $1.2 \pm 0.1$ & $3.2 \pm 0.3$ & $1.2 \pm 0.1$ & $0.17 \pm 0.01$ & $0.50 \pm 0.05$ & $0.21 \pm 0.02$ & $0.46 \pm 0.04$ \\
\hline
\end{tabular}

Table 7 (b)

\begin{tabular}{|c|c|c|c|c|c|c|c|c|c|c|}
\hline \multirow{3}{*}{$\begin{array}{l}\text { Health } \\
\text { centre }\end{array}$} & \multicolumn{5}{|l|}{${ }^{218} \mathrm{Po}$} & \multicolumn{5}{|l|}{${ }^{214} \mathrm{Po}$} \\
\hline & \multicolumn{3}{|c|}{ Thoracic region } & \multicolumn{2}{|c|}{ Extrathoracic region } & \multicolumn{3}{|c|}{ Thoracic region } & \multicolumn{2}{|c|}{ Extrathoracic region } \\
\hline & 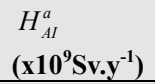 & $\begin{array}{l}H_{b b}^{a} \\
\left(\mathbf{x 1 0}^{7} \mathbf{S v} \cdot \mathbf{y}^{-1}\right)\end{array}$ & $\begin{array}{l}H_{B B}^{a} \\
\left(\mathbf{x 1 0}^{7} \mathbf{S v} \cdot \mathbf{y}^{-1}\right)\end{array}$ & $\begin{array}{l}H_{E T 2}^{a} \\
\left(\mathbf{x 1 0} \mathbf{S v}^{\mathbf{5}} \mathbf{\mathrm { y }} \mathbf{y}^{-1}\right)\end{array}$ & 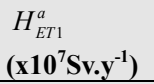 & $\begin{array}{l}H_{A I}^{a} \\
\left(\mathbf{x 1 0 ^ { 1 6 }} \mathbf{S v}^{\left.-\mathbf{y}^{-1}\right)}\right.\end{array}$ & $\begin{array}{l}H_{b b}^{a} \\
\left(\mathbf{x 1 0 ^ { 1 3 }} \mathbf{S v}^{-\mathbf{y}^{-1}}\right)\end{array}$ & 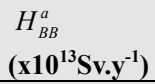 & $\begin{array}{l}H_{E T 2}^{a} \\
\left(\mathbf{x 1 0 ^ { 1 1 }} \mathbf{S v} \cdot \mathbf{y}^{-1}\right)\end{array}$ & 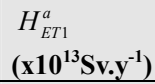 \\
\hline $\mathrm{HC} 1$ & $7.0 \pm 0.7$ & $8.4 \pm 0.8$ & $2.4 \pm 0.2$ & $0.90 \pm 0.08$ & $2.3 \pm 0.2$ & $38 \pm 3$ & $4.5 \pm 0.4$ & $1.3 \pm 0.1$ & $0.60 \pm 0.05$ & $1.2 \pm 0.1$ \\
\hline $\mathrm{HC} 2$ & $6.2 \pm 0.6$ & $7.5 \pm 0.7$ & $2.1 \pm 0.2$ & $0.80 \pm 0.07$ & $2.1 \pm 0.2$ & $32 \pm 3$ & $3.9 \pm 0.3$ & $1.1 \pm 0.1$ & $0.50 \pm 0.04$ & $1.1 \pm 0.1$ \\
\hline $\mathrm{HC} 3$ & $10 \pm 1$ & $12 \pm 1$ & $3.5 \pm 0.3$ & $1.3 \pm 0.1$ & $3.4 \pm 0.3$ & $60 \pm 5$ & $7.2 \pm 0.7$ & $2.0 \pm 0.2$ & $1.0 \pm 0.1$ & $2.0 \pm 0.2$ \\
\hline $\mathrm{HC} 4$ & $6.0 \pm 0.6$ & $7.2 \pm 0.7$ & $2.0 \pm 0.2$ & $0.80 \pm 0.07$ & $2.0 \pm 0.2$ & $30 \pm 3$ & $3.7 \pm 0.3$ & $1.0 \pm 0.1$ & $0.50 \pm 0.04$ & $1.0 \pm 0.1$ \\
\hline HC5 & $6.0 \pm 0.6$ & $7.2 \pm 0.7$ & $2.0 \pm 0.2$ & $0.80 \pm 0.07$ & $2.0 \pm 0.2$ & $30 \pm 3$ & $3.7 \pm 0.3$ & $1.0 \pm 0.1$ & $0.50 \pm 0.04$ & $1.0 \pm 0.1$ \\
\hline HC6 & $7.6 \pm 0.7$ & $9.1 \pm 0.8$ & $2.6 \pm 0.2$ & $1.0 \pm 0.1$ & $2.5 \pm 0.2$ & $42 \pm 4$ & $5.0 \pm 0.5$ & $1.4 \pm 0.1$ & $0.70 \pm 0.06$ & $1.4 \pm 0.1$ \\
\hline $\mathrm{HC} 7$ & $5.6 \pm 0.5$ & $6.8 \pm 0.6$ & $1.9 \pm 0.1$ & $0.70 \pm 0.06$ & $1.9 \pm 0.1$ & $27 \pm 2$ & $3.2 \pm 0.3$ & $0.9 . \pm 0.08$ & $0.40 \pm 0.03$ & $0.90 \pm 0.08$ \\
\hline $\mathrm{HC} 8$ & $5.7 \pm 0.5$ & $6.9 \pm 0.6$ & $2.0 \pm 0.2$ & $0.70 \pm 0.06$ & $1.9 \pm 0.1$ & $28 \pm 2$ & $3.4 \pm 0.3$ & $1.0 \pm 0.1$ & $0.50 \pm 04$ & $0.90 \pm 0.08$ \\
\hline $\mathrm{HC} 9$ & $10 \pm 1$ & $12 \pm 1$ & $3.5 \pm 0.3$ & $1.3 \pm 0.1$ & $3.4 \pm 0.3$ & $60 \pm 5$ & $7.2 \pm 0.7$ & $2.0 \pm 0.2$ & $1.0 \pm 0.1$ & $2.0 \pm 0.2$ \\
\hline $\mathrm{HC} 10$ & $6.4 \pm 0.6$ & $7.8 \pm 0.7$ & $2.2 \pm 0.2$ & $0.80 \pm 0.07$ & $2.1 \pm 0.2$ & $34 \pm 3$ & $4.1 \pm 0.4$ & $1.1 \pm 0.1$ & $0.50 \pm 0.04$ & $1.1 \pm 0.1$ \\
\hline
\end{tabular}

Table 8. Data obtained for the committed equivalent doses due to the unattached (a) and attached (b) fractions of the ${ }^{218}$ Po and ${ }^{214} P o$ radon progeny in the tissues of the respiratory tract from the inhalation of air by 5 y child in different health centres. Given errors correspond to one-sigma uncertainties.

Table 8 (a)

\begin{tabular}{|c|c|c|c|c|c|c|c|c|c|c|}
\hline \multirow{3}{*}{$\begin{array}{l}\text { Health } \\
\text { centre }\end{array}$} & \multicolumn{5}{|l|}{${ }^{218} \mathrm{Po}$} & \multicolumn{5}{|l|}{${ }^{214} \mathrm{Po}$} \\
\hline & \multicolumn{3}{|c|}{ Thoracic region } & \multicolumn{2}{|c|}{ Extrathoracic region } & \multicolumn{3}{|c|}{ Thoracic region } & \multicolumn{2}{|c|}{ Extrathoracic region } \\
\hline & $\begin{array}{l}H_{A I}^{u} \\
\left(\mathbf{x 1 0 ^ { 1 2 }} \mathbf{S v}^{\left.-\mathbf{y}^{-1}\right)}\right)\end{array}$ & $\begin{array}{l}H_{b b}^{u} \\
\left(\mathbf{x 1 0}^{7} \mathbf{S v} \cdot \mathbf{y}^{-1}\right)\end{array}$ & $\begin{array}{l}H_{B B}^{u} \\
\left(\mathbf{x 1 0}^{\mathbf{7}} \mathbf{S v} \cdot \mathbf{y}^{-1}\right) \\
\end{array}$ & $\begin{array}{l}H_{E T 2}^{u} \\
\left(\mathbf{x 1 0 ^ { 5 }} \mathbf{S v} \cdot \mathbf{y}^{-1}\right)\end{array}$ &  & $\begin{array}{l}H_{A I}^{u} \\
\left(\mathbf{x 1 0 ^ { 2 1 }} \mathbf{S v}_{\cdot} \mathbf{y}^{-1}\right)\end{array}$ & $\begin{array}{l}H_{b b}^{u} \\
\left(\mathbf{x 1 0 ^ { 1 5 }} \mathbf{S v} \cdot \mathbf{y}^{-1}\right)\end{array}$ & $\begin{array}{l}H_{B B}^{u} \\
\left(\mathbf{x 1 0}^{15} \mathbf{S v} \cdot \mathbf{y}^{-1}\right)\end{array}$ & $\begin{array}{l}H_{E T 2}^{u} \\
\left(\mathbf{x 1 0}{ }^{13} \mathbf{S v} \cdot \mathbf{y}^{-1}\right)\end{array}$ & 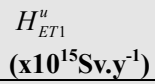 \\
\hline $\mathrm{HC} 1$ & $1.4 \pm 0.1$ & $1.4 \pm 0.1$ & $4.4 \pm 0.4$ & $1.6 \pm 0.1$ & $4.5 \pm 0.4$ & $2.2 \pm 0.2$ & $0.20 \pm 0.02$ & $0.60 \pm 0.05$ & $0.29 \pm 0.02$ & $0.70 \pm 0.06$ \\
\hline $\mathrm{HC} 2$ & $1.3 \pm 0.1$ & $1.2 \pm 0.1$ & $3.9 \pm 0.3$ & $1.4 \pm 0.1$ & $4.0 \pm 0.4$ & $1.9 \pm 0.1$ & $0.18 \pm 0.01$ & $0.60 \pm 0.05$ & $0.26 \pm 0.02$ & $0.60 \pm 0.05$ \\
\hline $\mathrm{HC} 3$ & $2.1 \pm 0.2$ & $2.0 \pm 0.2$ & $6.4 \pm 0.6$ & $2.3 \pm 0.2$ & $6.6 \pm 0.6$ & $3.2 \pm 0.3$ & $0.29 \pm 0.02$ & $0.90 \pm 0.08$ & $0.42 \pm 0.04$ & $1.0 \pm 0.1$ \\
\hline $\mathrm{HC} 4$ & $1.2 \pm 0.1$ & $1.2 \pm 0.1$ & $3.8 \pm 0.3$ & $1.4 \pm 0.1$ & $3.9 \pm 0.3$ & $1.9 \pm 0.1$ & $0.17 \pm 0.01$ & $0.60 \pm 0.05$ & $0.25 \pm 0.02$ & $0.60 \pm 0.05$ \\
\hline HC5 & $1.2 \pm 0.1$ & $1.2 \pm 0.1$ & $3.8 \pm 0.3$ & $1.4 \pm 0.1$ & $3.9 \pm 0.3$ & $1.9 \pm 0.1$ & $0.17 \pm 0.01$ & $0.60 \pm 0.05$ & $0.25 \pm 0.02$ & $0.60 \pm 0.05$ \\
\hline HC6 & $1.5 \pm 0.1$ & $1.5 \pm 0.1$ & $4.8 \pm 0.4$ & $1.7 \pm 0.1$ & $4.9 \pm 0.4$ & $2.4 \pm 0.2$ & $0.22 \pm 0.02$ & $0.70 \pm 0.06$ & $0.31 \pm 0.03$ & $0.70 \pm 0.06$ \\
\hline $\mathrm{HC7}$ & $1.2 \pm 0.1$ & $1.1 \pm 0.1$ & $3.6 \pm 0.3$ & $1.3 \pm 0.1$ & $3.7 \pm 0.3$ & $1.7 \pm 0.1$ & $0.16 \pm 0.01$ & $0.50 \pm 0.04$ & $0.23 \pm 0.02$ & $0.50 \pm 0.04$ \\
\hline $\mathrm{HC} 8$ & $1.2 \pm 0.1$ & $1.1 \pm 0.1$ & $3.7 \pm 0.3$ & $1.3 \pm 0.1$ & $3.8 \pm 0.3$ & $1.8 \pm 0.1$ & $0.17 \pm 0.01$ & $0.50 \pm 0.04$ & $0.24 \pm 0.02$ & $0.50 \pm 0.04$ \\
\hline HC9 & $2.1 \pm 0.2$ & $2.0 \pm 0.2$ & $6.4 \pm 0.6$ & $2.3 \pm 0.2$ & $6.6 \pm 0.5$ & $3.2 \pm 0.3$ & $0.30 \pm 0.03$ & $1.0 \pm 0.1$ & $0.42 \pm 0.04$ & $1.0 \pm 0.1$ \\
\hline $\mathrm{HC} 10$ & $1.3 \pm 0.1$ & $1.3 \pm 0.1$ & $4.1 \pm 0.4$ & $1.4 \pm 0.1$ & $4.2 \pm 0.4$ & $2.0 \pm 0.2$ & $0.19 \pm 0.01$ & $0.60 \pm 0.05$ & $0.27 \pm 002$ & $0.60 \pm 0.05$ \\
\hline
\end{tabular}


Table 8 (b)

\begin{tabular}{|c|c|c|c|c|c|c|c|c|c|c|}
\hline \multirow{3}{*}{$\begin{array}{l}\text { Health } \\
\text { centre }\end{array}$} & \multicolumn{5}{|l|}{${ }^{218} \mathrm{Po}$} & \multicolumn{5}{|l|}{${ }^{214} \mathrm{Po}$} \\
\hline & \multicolumn{2}{|c|}{ Thoracic region } & \multicolumn{3}{|c|}{ Extrathoracic region } & \multicolumn{2}{|c|}{ Thoracic region } & \multicolumn{3}{|c|}{ Extrathoracic region } \\
\hline & $\begin{array}{l}H_{A I}^{a} \\
\left(\mathbf{x 1 0}^{\mathbf{9}} \mathbf{S v} \cdot \mathbf{y}^{-1}\right)\end{array}$ & $\begin{array}{l}H_{b b}^{a} \\
\left(\mathbf{x 1 0} \mathbf{0}^{7} \mathbf{S v} \cdot \mathbf{y}^{-1}\right)\end{array}$ & $\begin{array}{l}H_{B B}^{a} \\
\left(\mathbf{x 1 0} \mathbf{0}^{7} \mathbf{S v} \cdot \mathbf{y}^{-1}\right) \\
\end{array}$ & $\begin{array}{l}H_{E T 2}^{a} \\
\left(\mathbf{x 1 0}^{\mathbf{5}} \mathbf{S v} \cdot \mathbf{y}^{-1}\right)\end{array}$ & $\begin{array}{l}H_{E T 1}^{a} \\
\left(\mathbf{x 1 0} \mathbf{S V}^{-1} \mathbf{y}^{-1}\right)\end{array}$ & $\begin{array}{l}H_{A I}^{a} \\
\left(\mathbf{x 1 0 ^ { 1 5 }} \mathbf{S v} \cdot \mathbf{y}^{-1}\right)\end{array}$ & $\begin{array}{l}H_{b b}^{a} \\
\left(\mathbf{x 1 0}{ }^{13} \mathbf{S v} \cdot \mathbf{y}^{-1}\right)\end{array}$ & $\begin{array}{l}H_{B B}^{a} \\
\left(\mathbf{x 1 0} 0^{13} \mathbf{S v} \cdot \mathbf{y}^{-1}\right)\end{array}$ & $\begin{array}{l}H_{E T 2}^{a} \\
\left(\mathbf{x 1 0}^{11} \mathbf{S v} \cdot \mathbf{y}^{-1}\right) \\
\end{array}$ & $\begin{array}{l}H_{E T 1}^{a} \\
\left(\mathbf{x 1 0 ^ { 1 3 }} \mathbf{S v} \cdot \mathbf{y}^{-1}\right)\end{array}$ \\
\hline CS1 & $11 \pm 1$ & $9.3 \pm 0.8$ & $2.8 \pm 0.2$ & $1.5 \pm 0.1$ & $3.9 \pm 0.3$ & $6.0 \pm 0.6$ & $5.0 \pm 0.5$ & $1.5 \pm 0.1$ & $1.0 \pm 0.1$ & $2.1 \pm 0.2$ \\
\hline $\mathrm{CS} 2$ & $10 \pm 1$ & $8.3 \pm 0.8$ & $2.5 \pm 0.2$ & $1.3 \pm 0.1$ & $3.5 \pm 0.3$ & $5.1 \pm 0.5$ & $4.3 \pm 0.4$ & $1.3 \pm 0.1$ & $0.90 \pm 0.08$ & $1.8 \pm 0.1$ \\
\hline $\mathrm{CS} 3$ & $16 \pm 1$ & $14 \pm 1$ & $4.1 \pm 0.4$ & $2.2 \pm 0.2$ & $5.7 \pm 0.5$ & $9.6 \pm 0.8$ & $7.9 \pm 0.7$ & $2.4 \pm 0.2$ & $1.6 \pm 0.1$ & $3.3 \pm 0.3$ \\
\hline CS4 & $9.7 \pm 0.8$ & $8.0 \pm 0.8$ & $2.4 \pm 0.2$ & $1.3 \pm 0.1$ & $3.3 \pm 0.3$ & $4.9 \pm 0.4$ & $4.0 \pm 0.4$ & $1.2 \pm 0.1$ & $0.80 \pm 0.07$ & $1.7 \pm 0.1$ \\
\hline CS5 & $9.7 \pm 0.8$ & $8.0 \pm 0.8$ & $2.4 \pm 0.2$ & $1.3 \pm 0.1$ & $3.3 \pm 0.3$ & $4.9 \pm 0.4$ & $4.0 \pm 0.4$ & $1.2 \pm 0.1$ & $0.80 \pm 0.07$ & $1.7 \pm 0.1$ \\
\hline CS6 & $12 \pm 1$ & $10 \pm 1$ & $3.0 \pm 0.3$ & $1.6 \pm 0.1$ & $4.2 \pm 0.4$ & $6.7 \pm 0.6$ & $5.5 \pm 0.5$ & $1.7 \pm 0.1$ & $1.1 \pm 0.1$ & $2.3 \pm 0.2$ \\
\hline CS7 & $9.0 \pm 0.8$ & $7.5 \pm 0.7$ & $2.2 \pm 0.2$ & $1.2 \pm 0.1$ & $3.1 \pm 0.3$ & $4.3 \pm 0.4$ & $3.5 \pm 0.3$ & $1.1 \pm 0.1$ & $0.70 \pm 0.06$ & $1.5 \pm 0.1$ \\
\hline CS8 & $9.3 \pm 0.8$ & $7.7 \pm 0.7$ & $2.3 \pm 0.2$ & $1.2 \pm 0.1$ & $3.2 \pm 0.3$ & $4.5 \pm 0.4$ & $3.7 \pm 0.3$ & $1.1 \pm 0.1$ & $0.80 \pm 0.07$ & $1.6 \pm 0.1$ \\
\hline CS9 & $16 \pm 1$ & $14 \pm 1$ & $4.1 \pm 0.4$ & $2.2 \pm 0.2$ & $5.7 \pm 0.5$ & $9.6 \pm 0.8$ & $8.0 \pm 0.8$ & $2.4 \pm 0.2$ & $1.6 \pm 0.1$ & $3.3 \pm 0.3$ \\
\hline CS10 & $10 \pm 1$ & $8.6 \pm 0.7$ & $2.6 \pm 0.2$ & $1.4 \pm 0.1$ & $3.6 \pm 0.3$ & $5.5 \pm 0.5$ & $4.5 \pm 0.4$ & $1.3 \pm 0.1$ & $0.90 \pm 0.08$ & $1.9 \pm 0.1$ \\
\hline
\end{tabular}

After inhalation of air, ${ }^{218} \mathrm{Po}$ and ${ }^{214} \mathrm{Po}$ are deposited in the extrathoracic region ET and thoracic region TH of the lung. According to the ICRP compartmental model [16] a certain number of ${ }^{218} \mathrm{Po}$ atoms are transferred from the $\mathrm{TH}$ region to the ET region, since this radionuclide has a half-life of $3.05 \mathrm{~min}$. As a consequence, we found that annual committed equivalent doses due to ${ }^{218} \mathrm{Po}$ (attached and unattached fractions) are higher in the ET region than in the TH region for doctors, nurses and patients (Tables 4-8). It is noted that annual committed equivalent doses due to ${ }^{214} \mathrm{Po}$ (attached and unattached fractions) are smaller than those due to ${ }^{218} \mathrm{Po}$ in both ET and TH regions. This is due to fact that ${ }^{214} \mathrm{Po}$ has a very short half-life $\left(1.6410^{-4} \mathrm{~s}\right)$ compared to the exposure time of the tissues: this means that ${ }^{214} \mathrm{Po}$ comes essentially from the disintegration of ${ }^{218} \mathrm{Po}$ present in the ET and $\mathrm{TH}$ regions.

$$
H_{T}^{a}\left({ }^{218} \mathrm{Po}\right), H_{T}^{u}\left({ }^{218} \mathrm{Po}\right), H_{T}^{a}\left({ }^{214} \mathrm{Po}\right) \text { and } H_{T}^{u}\left({ }^{214} \mathrm{Po}\right) \text { are }
$$
influenced by the integral of activity-time curves (the activity integrals)(Equations (9) and (12)) of ${ }^{218} \mathrm{Po}$ and ${ }^{214} \mathrm{Po}$ in a given tissue of the human body, the mass of the target tissue $\left(\mathrm{m}_{\mathrm{T}}\right)$ and the weighting factor for the partition of radiation detriment (Equations(13) and (14)). Variation of the activities of ${ }^{218} \mathrm{Po}$ and ${ }^{214} \mathrm{Po}$ as functions of time in various tissues of the human body from the inhalation of air by different age groups of individuals are given in reference [27]

The results in Tables 4-8 show that:

- $H_{T}^{a}\left({ }^{218} \mathrm{Po}\right), H_{T}^{u}\left({ }^{218} \mathrm{Po}\right), H_{T}^{a}\left({ }^{214} \mathrm{Po}\right)$ and $H_{T}^{u}\left({ }^{214} \mathrm{Po}\right)$ are clearly higher in the bb and $\mathrm{BB}$ tissues than in the AI tissue of the thoracic region even though the latter tissue shows higher activity integral than the former ones[27]. This is due to the predominance of the mass tissue: the former tissues show lower masses than the latter.

- $H_{T}^{a}\left({ }^{218} \mathrm{Po}\right), H_{T}^{u}\left({ }^{218} \mathrm{Po}\right), H_{T}^{a}\left({ }^{214} \mathrm{Po}\right)$ and $H_{T}^{u}\left({ }^{214} \mathrm{Po}\right)$ are higher in the $\mathrm{ET}_{2}$ tissue than in the $\mathrm{ET}_{1}$ tissue of the extrathoracic region even though the latter tissue has smaller mass and higher activity integral than the former one. This is because the former tissue shows a higher weighting factor for the partition of radiation detriment than the latter.

- $H_{T}^{a}\left({ }^{218} \mathrm{Po}\right), H_{T}^{u}\left({ }^{218} \mathrm{Po}\right), H_{T}^{a}\left({ }^{214} \mathrm{Po}\right)$ and $H_{T}^{u}\left({ }^{214} \mathrm{Po}\right)$ in the $\mathrm{bb}$ tissue are higher for the adult male than for the adult female: it is due to the predominance of the activity integral [27].

$$
\text { - } H_{T}^{a}\left({ }^{218} \mathrm{Po}\right), H_{T}^{u}\left({ }^{218} \mathrm{Po}\right), H_{T}^{a}\left({ }^{214} \mathrm{Po}\right) \text { and } H_{T}^{u}\left({ }^{214} \mathrm{Po}\right) \text { are }
$$
negligible in the $\mathrm{LN}_{\mathrm{TH}}$ (thoracic region) and $\mathrm{LN}_{\mathrm{ET}}$ (extrathoracic region) tissues. This is due to the fact that these tissues show lower activity integrals and weighting factors for the partition of radiation detriment.

Committed effective doses per hour of exposure due to the attached $E^{a}$ and unattached $E^{u}$ fractions of the ${ }^{218} \mathrm{Po}$ and ${ }^{214}$ Po radon decay products and global committed effective dose $E$ from the inhalation of air by doctors, nurses and patients in different health centres were evaluated by use of Equations 15-17. Data obtained are shown in Tables 9 and 10.The statistical relative uncertainty of the committed effective dose determination is about $10 \%$. It was noted that the global committed effective dose due to ${ }^{218} \mathrm{Po}$ and ${ }^{214} \mathrm{Po}$ radon progeny is higher for patients in the HC3 and HC9 health centres than in the others (Table 10). This is because activities due to these radionuclides are higher in these locations than in the others (Table 3(c)). It was also noted that global committed effective dose due to ${ }^{218} \mathrm{Po}$ and ${ }^{214} \mathrm{Po}$ is higher for $5 y$ child than for the other age groups of patients. This is due to the fact that $5 \mathrm{y}$ child have smaller lung mass than the others age groups. A maximum value of $7.1 \mathrm{mSv} \mathrm{y}^{-1}$ was found for female doctors spending 8 hours per day (2,080 hours per year) in the HC4 health centre which is within the (3-10 $\left.\mathrm{mSvy}^{-1}\right)$ dose limit interval for workers [21]. 
149 M. A. Misdaq et al:: Radon, Thoron and Progeny Measured in Urban Health Centres and the Resulting Radiation Doses to Doctors, Nurses and Patients from the Inhalation of Air

Table 9. Data obtained for the committed dose per hour of exposure due to the attached $E^{a}$ and unattached $E^{u}$ fractions of the ${ }^{218}$ Po and ${ }^{214}$ Po radon decay products and global committed effective dose E from the inhalation of air by doctors and nurses in different health centres.

\begin{tabular}{|c|c|c|c|c|c|c|c|c|c|c|c|c|}
\hline \multirow{4}{*}{$\begin{array}{l}\text { Health } \\
\text { center }\end{array}$} & \multicolumn{12}{|c|}{ Committed effective dose ( $\mu \mathrm{Sv} \mathrm{y}^{-1} \mathrm{~h}^{-1}$ exposure) } \\
\hline & \multicolumn{6}{|l|}{ Doctors } & \multicolumn{6}{|l|}{ Nurses } \\
\hline & \multicolumn{3}{|l|}{ Male } & \multicolumn{3}{|l|}{ Female } & \multicolumn{3}{|l|}{ Male } & \multicolumn{3}{|l|}{ Female } \\
\hline & $E^{u}$ & $E^{a}$ & $E$ & $E^{u}$ & $E^{a}$ & $E$ & $E^{u}$ & $E^{a}$ & $E$ & $E^{u}$ & $E^{a}$ & E \\
\hline $\mathrm{HC} 1$ & $0.60 \pm 0.05$ & $0.70 \pm 0.06$ & $1.4 \pm 0.1$ & $0.70 \pm 0.06$ & $0.80 \pm 0.07$ & $1.5 \pm 0.1$ & $0.70 \pm 0.06$ & $0.80 \pm 0.07$ & $1.4 \pm 0.1$ & $0.80 \pm 0.07$ & $0.90 \pm 0.08$ & $1.6 \pm 0.1$ \\
\hline $\mathrm{HC} 2$ & $0.6 \pm 0.05$ & $0.70 \pm 0.06$ & $1.4 \pm 0.1$ & $0.70 \pm 0.06$ & $0.80 \pm 0.07$ & $1.5 \pm 0.1$ & $0.70 \pm 0.06$ & $0.80 \pm 0.07$ & $1.4 \pm 0.1$ & $0.80 \pm 0.07$ & $0.80 \pm 0.07$ & $1.6 \pm 0.1$ \\
\hline $\mathrm{HC} 3$ & $1.1 \pm 0.1$ & $1.3 \pm 0.1$ & $2.4 \pm 0.2$ & $1.3 \pm 0.1$ & $1.4 \pm 0.1$ & $2.7 \pm 0.2$ & $1.2 \pm 0.1$ & $1.3 \pm 0.1$ & $2.5 \pm 0.2$ & $1.4 \pm 0.1$ & $1.5 \pm 0.1$ & $2.9 \pm 0.2$ \\
\hline $\mathrm{HC} 4$ & $1.4 \pm 0.1$ & $1.6 \pm 0.1$ & $3.0 \pm 0.3$ & $1.6 \pm 0.1$ & $1.8 \pm 0.1$ & $3.4 \pm 0.3$ & $0.80 \pm 0.07$ & $0.90 \pm 0.08$ & $1.6 \pm 0.1$ & $0.90 \pm 0.08$ & $1.0 \pm 0.1$ & $1.8 \pm 0.1$ \\
\hline HC5 & $1.1 \pm 0.1$ & $1.2 \pm 0.1$ & $2.3 \pm 0.2$ & $1.2 \pm 0.1$ & $1.4 \pm 0.1$ & $2.6 \pm 0.2$ & $0.80 \pm 0.07$ & $0.90 \pm 0.08$ & $1.7 \pm 0.1$ & $0.90 \pm 0.08$ & $1.0 \pm 0.1$ & $1.9 \pm 0.1$ \\
\hline HC6 & $1.0 \pm 0.1$ & $1.2 \pm 0.1$ & $2.2 \pm 0.2$ & $1.2 \pm 0.1$ & $1.3 \pm 0.1$ & $2.5 \pm 0.2$ & $0.90 \pm 0.08$ & $1.0 \pm 0.1$ & $1.9 \pm 0.1$ & $1.0 \pm 0.1$ & $1.1 \pm 0.1$ & $2.2 \pm 0.2$ \\
\hline $\mathrm{HC7}$ & $0.70 \pm 0.06$ & $0.70 \pm 0.06$ & $1.4 \pm 0.1$ & $0.80 \pm 0.07$ & $0.80 \pm 0.07$ & $1.6 \pm 0.1$ & $0.70 \pm 0.06$ & $0.80 \pm 0.07$ & $1.5 \pm 0.1$ & $0.80 \pm 0.07$ & $0.90 \pm 0.08$ & $1.6 \pm 0.1$ \\
\hline $\mathrm{HC} 8$ & $0.70 \pm 0.06$ & $0.80 \pm 0.07$ & $1.5 \pm 0.1$ & $0.80 \pm 0.07$ & $0.90 \pm 0.08$ & $1.7 \pm 0.1$ & $0.70 \pm 0.06$ & $0.80 \pm 0.07$ & $1.4 \pm 0.1$ & $0.80 \pm 0.07$ & $0.90 \pm 0.08$ & $1.6 \pm 0.1$ \\
\hline HC9 & $1.3 \pm 0.1$ & $1.5 \pm 0.1$ & $2.8 \pm 0.2$ & $1.5 \pm 0.1$ & $1.7 \pm 0.1$ & $3.2 \pm 0.3$ & $1.2 \pm 0.1$ & $1.4 \pm 0.1$ & $2.6 \pm 0.2$ & $1.4 \pm 0.1$ & $1.5 \pm 0.1$ & $2.9 \pm 0.2$ \\
\hline $\mathrm{HC} 10$ & $0.90 \pm 0.08$ & $1.0 \pm 0.1$ & $1.8 \pm 0.1$ & $1.0 \pm 0.1$ & $1.1 \pm 0.1$ & $2.0 \pm 0.2$ & $0.80 \pm 0.07$ & $0.90 \pm 0.08$ & $1.6 \pm 0.1$ & $0.90 \pm 0.08$ & $1.0 \pm 0.1$ & $1.8 \pm 0.1$ \\
\hline
\end{tabular}

Table 10. Data obtained for the committed effective doses per hour of exposure due to the attached $E^{a}$ and unattached $E^{u}$ fractions of the ${ }^{218} P o$ and ${ }^{214} P o$ radon decay products and global committed effective dose E from the inhalation of air by different age groups of patients in various health centres.

\begin{tabular}{|c|c|c|c|c|c|c|c|c|c|}
\hline \multirow{3}{*}{ Health centre } & \multicolumn{9}{|c|}{ Committed effective dose ( $\mu \mathrm{Sv} \mathrm{y}^{-1} \mathrm{~h}^{-1}$ exposure) } \\
\hline & \multicolumn{3}{|l|}{ Adult male } & \multicolumn{3}{|c|}{ Adult female } & \multicolumn{3}{|c|}{ Male, 15 years } \\
\hline & $E^{u}$ & $E^{a}$ & $E$ & $E^{u}$ & $E^{a}$ & E & $E^{u}$ & $E^{a}$ & E \\
\hline $\mathrm{HC} 1$ & $0.35 \pm 0.03$ & $0.29 \pm 0.02$ & $0.64 \pm 0.06$ & $0.28 \pm 0.02$ & $0.24 \pm 0.02$ & $0.52 \pm 0.05$ & $0.31 \pm 0.03$ & $0.26 \pm 0.02$ & $0.57 \pm 0.05$ \\
\hline $\mathrm{HC} 2$ & $0.31 \pm 0.03$ & $0.26 \pm 0.02$ & $0.57 \pm 0.05$ & $0.25 \pm 0.02$ & $0.21 \pm 0.02$ & $0.46 \pm 0.04$ & $0.27 \pm 0.02$ & $0.23 \pm 0.02$ & $0.50 \pm 0.05$ \\
\hline $\mathrm{HC} 3$ & $0.51 \pm 0.05$ & $0.42 \pm 0.04$ & $0.93 \pm 0.09$ & $0.41 \pm 0.04$ & $0.35 \pm 0.03$ & $0.76 \pm 0.07$ & $0.45 \pm 0.04$ & $0.38 \pm 0.03$ & $0.83 \pm 0.08$ \\
\hline $\mathrm{HC} 4$ & $0.30 \pm 0.03$ & $0.25 \pm 0.02$ & $0.55 \pm 0.05$ & $0.25 \pm 0.02$ & $0.20 \pm 0.02$ & $0.45 \pm 0.04$ & $0.27 \pm 0.02$ & $0.22 \pm 0.02$ & $0.49 \pm 0.04$ \\
\hline HC5 & $0.30 \pm 0.03$ & $0.25 \pm 0.02$ & $0.55 \pm 0.05$ & $0.25 \pm 0.02$ & $0.20 \pm 0.02$ & $0.45 \pm 0.04$ & $0.27 \pm 0.02$ & $0.22 \pm 0.02$ & $0.49 \pm 0.04$ \\
\hline HC6 & $0.38 \pm 0.03$ & $0.31 \pm 0.03$ & $0.69 \pm 0.06$ & $0.31 \pm 0.03$ & $0.26 \pm 0.02$ & $0.57 \pm 0.05$ & $0.33 \pm 0.03$ & $0.28 \pm 0.02$ & $0.61 \pm 0.06$ \\
\hline $\mathrm{HC} 7$ & $0.28 \pm 0.02$ & $0.23 \pm 0.02$ & $0.51 \pm 0.05$ & $0.23 \pm 0.02$ & $0.19 \pm 0.01$ & $0.42 \pm 0.04$ & $0.25 \pm 0.02$ & $0.21 \pm 0.02$ & $0.46 \pm 0.04$ \\
\hline $\mathrm{HC} 8$ & $0.29 \pm 0.02$ & $0.24 \pm 0.02$ & $0.53 \pm 0.05$ & $0.24 \pm 0.02$ & $0.19 \pm 0.01$ & $0.43 \pm 0.04$ & $0.25 \pm 0.02$ & $0.21 \pm 0.02$ & $0.46 \pm 0.04$ \\
\hline HC9 & $0.51 \pm 0.05$ & $0.42 \pm 0.04$ & $0.93 \pm 0.08$ & $0.41 \pm 0.04$ & $0.35 \pm 0.03$ & $0.76 \pm 0.07$ & $0.45 \pm 0.04$ & $0.38 \pm 0.03$ & $0.83 \pm 0.08$ \\
\hline HC10 & $0.32 \pm 0.03$ & $0.27 \pm 0.02$ & $0.59 \pm 0.05$ & $0.26 \pm 0.02$ & $0.22 \pm 0.02$ & $0.48 \pm 0.04$ & $0.28 \pm 0.02$ & $0.24 \pm 0.02$ & $0.52 \pm 0.05$ \\
\hline
\end{tabular}

Table 10. Continue.

\begin{tabular}{|c|c|c|c|c|c|c|c|c|c|}
\hline \multirow{3}{*}{ Health centre } & \multicolumn{9}{|c|}{ Committed effective dose ( $\mu S v y^{-1} h^{-1}$ exposure) } \\
\hline & \multicolumn{3}{|c|}{ Female, 15years } & \multicolumn{3}{|c|}{ Chid, 10 years } & \multicolumn{3}{|c|}{ Chid, 5 years } \\
\hline & $E^{u}$ & $E^{a}$ & $E$ & $E^{u}$ & $E^{a}$ & $E$ & $E^{u}$ & $E^{a}$ & $E$ \\
\hline $\mathrm{HC} 1$ & $0.29 \pm 0.02$ & $0.24 \pm 0.02$ & $0.53 \pm 0.05$ & $0.38 \pm 0.03$ & $0.36 \pm 0.03$ & $0.74 \pm 0.07$ & $0.47 \pm 0.04$ & $0.53 \pm 0.05$ & $1.0 . \pm 0.1$ \\
\hline $\mathrm{HC} 2$ & $0.26 \pm 0.02$ & $0.21 \pm 0.02$ & $0.47 \pm 0.04$ & $0.34 \pm 0.03$ & $0.32 \pm 0.03$ & $0.66 \pm 0.06$ & $0.42 \pm 0.04$ & $0.47 \pm 0.04$ & $0.90 \pm 0.09$ \\
\hline $\mathrm{HC} 3$ & $0.42 \pm 0.04$ & $0.35 \pm 0.03$ & $0.77 \pm 0.07$ & $0.56 \pm 0.05$ & $0.53 \pm 0.05$ & $1.1 \pm 0.1$ & $0.69 \pm 0.06$ & $0.78 \pm 0.07$ & $1.5 \pm 0.1$ \\
\hline $\mathrm{HC} 4$ & $0.25 \pm 0.02$ & $0.20 \pm 0.02$ & $0.45 \pm 0.04$ & $0.33 \pm 0.03$ & $0.31 \pm 0.03$ & $0.64 \pm 0.06$ & $0.41 \pm 0.04$ & $0.46 \pm 0.04$ & $0.87 \pm 0.08$ \\
\hline HC5 & $0.25 \pm 0.02$ & $0.20 \pm 0.02$ & $0.45 \pm 0.04$ & $0.33 \pm 0.03$ & $0.31 \pm 0.03$ & $0.64 \pm 0.06$ & $0.41 \pm 0.04$ & $0.46 \pm 0.04$ & $0.90 \pm 0.09$ \\
\hline HC6 & $0.32 \pm 0.03$ & $0.26 \pm 0.03$ & $0.58 \pm 0.05$ & $0.41 \pm 0.04$ & $0.39 \pm 0.03$ & $0.80 \pm 0.08$ & $0.51 \pm 0.05$ & $0.57 \pm 0.05$ & $1.1 \pm 0.1$ \\
\hline $\mathrm{HC} 7$ & $0.24 \pm 0.02$ & $0.19 \pm 0.01$ & $0.43 \pm 0.04$ & $0.31 \pm 0.03$ & $0.29 \pm 0.02$ & $0.60 \pm 0.06$ & $0.38 \pm 0.03$ & $0.42 \pm 0.04$ & $0.80 \pm 0.08$ \\
\hline $\mathrm{HC} 8$ & $0.24 \pm 0.02$ & $0.19 \pm 0.01$ & $0.43 \pm 0.04$ & $0.32 \pm 0.03$ & $0.29 \pm 0.02$ & $0.61 \pm 0.06$ & $0.39 \pm 0.03$ & $0.44 \pm 0.04$ & $0.83 \pm 0.08$ \\
\hline HC9 & $0.43 \pm 0.04$ & $0.35 \pm 0.03$ & $0.78 \pm 0.07$ & $0.56 \pm 0.05$ & $0.53 \pm 0.05$ & $1.1 \pm 0.1$ & $0.69 \pm 0.06$ & $0.78 \pm 0.07$ & $1.5 \pm 0.1$ \\
\hline HC10 & $0.27 \pm 0.02$ & $0.22 \pm 0.02$ & $0.49 \pm 0.04$ & $0.35 \pm 0.03$ & $0.33 \pm 0.03$ & $0.68 \pm 0.06$ & $0.44 \pm 0.04$ & $0.49 \pm 0.04$ & $0.93 \pm 0.09$ \\
\hline
\end{tabular}


Committed effective doses due to ${ }^{222} \mathrm{Rn}$ progeny from the inhalation of air by patients (at rest) in the studied health centres were evaluated by using this model and the ICRP [18] conversion dose coefficient for the members of the general public $\left(10.5\left(\left(\mathrm{nSvh}^{-1}\right)\left(\mathrm{Bqm}^{-3}\right)\right)\right)$. Data obtained for the average effective dose obtained by using this model for adult patients (at rest) were found in good agreement with those obtained by using the

ICRP model for the general public (Table 11).

Table 11. Global committed effective dose ( $\mu S v y^{-1} h^{-1}$ of exposure)due to the attached and unattached fractions of the ${ }^{218} \mathrm{Po}$ and ${ }^{214} \mathrm{Po}$ radon short-lived progeny fromthe inhalation of air by adult patients inside different health centres by using the method described here and the ICRP dose conversion coefficient (18).

\begin{tabular}{lllll}
\hline \multirow{2}{*}{ Health centre } & \multicolumn{3}{l}{ Committed effective dose $\left(\boldsymbol{\mu S v} \mathbf{~}^{-1} \mathbf{h}^{-1}\right.$ of exposure $)$} \\
\cline { 2 - 4 } & \multicolumn{3}{l}{ This method } & \multirow{2}{*}{ ICRP } \\
\cline { 2 - 4 } & Adult male & Adultfemale & Average & \\
\hline HC1 & $0.64 \pm 0.05$ & $0.52 \pm 0.05$ & $0.58 \pm 0.05$ & 0.46 \\
HC2 & $0.57 \pm 0.05$ & $0.46 \pm 0.04$ & $0.51 \pm 0.05$ & 0.40 \\
HC3 & $0.93 \pm 0.08$ & $0.76 \pm 0.07$ & $0.84 \pm 0.08$ & 0.71 \\
HC4 & $0.55 \pm 0.05$ & $0.45 \pm 0.04$ & $0.50 \pm 0.05$ & 0.38 \\
HC5 & $0.55 \pm 0.05$ & $0.45 \pm 0.04$ & $0.50 \pm 0.05$ & 0.38 \\
HC6 & $0.69 \pm 0.05$ & $0.57 \pm 0.05$ & $0.63 \pm 0.06$ & 0.51 \\
HC7 & $0.51 \pm 0.05$ & $0.42 \pm 0.04$ & $0.46 \pm 0.04$ & 0.34 \\
HC8 & $0.53 \pm 0.05$ & $0.43 \pm 0.04$ & $0.48 \pm 0.04$ & 0.36 \\
HC9 & $0.93 \pm 0.09$ & $0.76 \pm 0.07$ & $0.84 \pm 0.08$ & 0.71 \\
HC10 & $0.59 \pm 0.05$ & $0.48 \pm 0.04$ & $0.53 \pm 0.05$ & 0.42 \\
\hline
\end{tabular}

\section{Conclusion}

It has been shown by this study that by using CR-39 and LR-115 type II solid state nuclear track detectors (SSNTDs) one can evaluate alpha-activities per unit volume due to ${ }^{222} \mathrm{Rn}$, ${ }^{220} \mathrm{Rn}$ and their decay products in the air of different health centres. Radiation doses to the respiratory tract of doctors, nurses and patients from the inhalation of air in the studied health centres were evaluated. It is concluded that global committed equivalent dose per hour of exposure due to the attached and unattached fractions of the ${ }^{218} \mathrm{Po}$ and ${ }^{214} \mathrm{Po}$ radon short-lived decay products increase when alphaactivities due to these radionuclides increase and the mass tissue decreases. Annual committed effective doses due ${ }^{218}$ Po and ${ }^{214} \mathrm{Po}$ radon short-lived progeny from the inhalation of air by working personnel and patients in the studied health centres were determined. It has been shown that the working personnel must spend less than 8 hours per day inside the studied health centres to avoid higher radiation dose exposure. Dose conversion coefficients obtained by using this model were found in good agreement with those of the ICRP for the inhalation of ${ }^{222} \mathrm{Rn}$ short-lived decay products. The SSNTD's method developed which has the advantage of being inexpensive, accurate, and sensitive and does not need the use of standard sources for its calibration is a good tool for measuring ${ }^{222} \mathrm{Rn},{ }^{220} \mathrm{Rn}$ and their progenies in the air of workplaces.

\section{Funding}

This work was performed under an URAC-15 research contract with the CNRST, Rabat, Morocco.

\section{References}

[1] M.A. Misdaq, H. Ezzahery, D. Elbboubi,"Determination of equivalent dose rates and committed effective doses in the respiratory system from the inhalation of radon decay products by using SSNTD and a dosimetric compartmental model”, Radiat. Prot. Dosim., 2001, Vol. 93, No. 4, pp347-355.

[2] M.A. Misdaq, K. Flata,"The influence of the cigarette smoke pollution and ventilation rate on alpha-activities per unit volume due to radon and its progeny",J. Environ. Radioactivity, 2003, Vol. 67, pp207-218.

[3] M.A. Misdaq, M. Ghilane, J. Ouguidi, K. Outeqablit,"Radiation doses to individuals due to $238 \mathrm{U}, 232 \mathrm{Th}$ and $222 \mathrm{Rn}$ from the immersion in thermal waters and to radon progeny from the inhalation of air inside thermal stations", Radiat. Environ. Biophysics, 2012, Vol. 51, pp375-389.

[4] K.N. Yu, E.C.M. Young, M.J. Stokes, Z.J. Guan, K.W. Cho, "A survey of radon and thoron progeny for dwellings in Hong Kong”, Health Phys.,1997,Vol. 73, No.2,pp373-377.

[5] C.M. Ha, S.Y. Chang, B. H. Lee,"Dose assessment to inhalation exposure of indoor ${ }^{222} \mathrm{Rn}$ daughters in Korea", Health Phys.,1999, Vol. 63, No. 4,pp453-456.

[6] United Nations Scientific Committee on the Effects of Atomic Radiation (UNSCEAR), Sources and effects of ionising radiation. Report to the General Assembly, New York, 1993.

[7] B. Lévesque, D. Gauvin, R.G. McGregor, R. Martel, S. Gingras, A. Dontigny A,"Radon in residences: Influence of geological and housing characteristics", Health Phys., 1997,Vol. 72, No. 6,pp907-914.

[8] N. Kávási, T. Kovács, C. Németh, T. Szabó, Z. Gorjánácz, A. Várhegyi A, "Difficulties in radon measurements at workplaces”, Radiat. Meas., 2006,Vol. 41,pp229-234.

[9] T. Anastasiou, H. Tsertos, S. Christofides, G. Christodoulides, "Indoor radon (222Rn) concentration measurements in Cyprus using high-sensitivity portable detectors",J. Environ. Radioactivity, 2003, Vol. 68, pp159-169.

[10] M.A. Misdaq, H. Khajmi, F. Aitnouh, S. Berrazzouk, W. Bourzik, "A new method for evaluating uranium and thorium contents in different natural material samples by calculating the CR-39 and LR-115 type II SSNTD detection efficiencies for the emitted $\alpha$-particles", Nucl. Instr. Meth. Phys. Res., 2000, Vol. B 171, pp350-359.

[11] A.F. Hafez, M.A. Naim,"Plastic nuclear track detection methods for estimation of thorium to uranium ratio in thick natural materials",Nucl. Instr. Meth. Phys. Res., 1992, Vol. 69B, pp373-381.

[12] M.A. Misdaq, A. Bakhchi, A. Ktata, A. Merzouki, N. Youbi, "Determination of uranium and thorium contents inside different materials using track detectors and mean critical angles", Appl. Radiat. Isot., 1999, Vol. 51,pp209-215. 
151 M. A. Misdaq et al:: Radon, Thoron and Progeny Measured in Urban Health Centres and the Resulting Radiation Doses to Doctors, Nurses and Patients from the Inhalation of Air

[13] M.A. Misdaq, H. Ezzahery, J. Lamine,"Influence of the building material and ventilation rate on the concentration of radon, thoron and their progenies in dwelling rooms using SSNTD and Monte Carlo simulation" J. Radioanal. Nucl. Chem., 2002, Vol.252, pp67-74.

[14] J. Porstendörfer, "Properties and behaviour of radon and thoron and their decay products in the air" J. Aerosol Sci.,1994, Vol.25, No. 2,pp219-263.

[15] J. Porstendörfer, T. T. Mercer, "Influence of nuclei concentration and humidity upon the attachment rate of atoms in the atmosphere" Atmos. Environ., 1978, Vol. 12,pp22232238 .

[16] International Commission on Radiological Protection (ICRP), Human respiratory tract model for radiological protection, ICRP Publication 66, Ann ICRP 24 (1-3), 1994.

[17] International Commission on Radiological Protection (ICRP), Human respiratory tract model for radiological protection, ICRP Publication 100, Ann ICRP 36 (1-2), 2006.

[18] International Commission on Radiological Protection (ICRP), Recommendations of the International Commission on Radiological Protection, ICRP Publication 103, Ann ICRP 37(2-4), 2007.

[19] J. P. Biersack, J.F. Ziegler, IBM Research, TRIM, Version 98, 1998.

[20] International Commission on Radiological Protection (ICRP), Basic anatomical and physiological data for use in radiological protection: reference values, ICRP Publication 89 , Ann ICRP 32 (3.4), 2002.
[21] International Commission on Radiological Protection, Protection against radon-222 at home and at work,ICRP Publication 65, Ann ICRP 23 (2), 1993.

[22] European Commission, Office for Official Publications of the European Commission, Recommendations for the implementation of the Title VII of the European Basic Safety Standards concerning significant increase due to natural radiation sources, Radiation Protection Series, 1997.

[23] D.W. Dixon, T.D. Gooding, S. Mc Cready Shea, "Evaluation and significance of radon exposure in British workplace buildings", Environ. Internat., 1996, Vol. 22, S1079-S1082.

[24] US EPA Environments Division (6609J), A Citizen's Guide to Radon: the Guide to Protecting Yourself and Your Family From Radon, US EPA 402-K-006, Washington DC,2004.

[25] G. Ákerblom, "Radon Legislation and National Guidelines", Swedish Radiation Protection Institute, SSI Report 99-18, ISSN 0282-4434, 1999.

[26] EU, Directive 2013/59/EURATOM, Laying down basic safety standards for protection against the dangers arising from exposure to ionising radiation, 2013.

[27] M. A. Misdaq, J.Ouguidi, "Concentrations of radon, thoron and their decay products measured in natural caves and ancient mines by using solid state nuclear track detectors and resulting radiation dose to the members of the public", J. Radioanal. Nucl. Chem., 2011, Vol.287, 135-150. 